\title{
WWP1 inactivation enhances efficacy of PI3K inhibitors while suppressing their toxicities in breast cancer models
}

\author{
Takahiro Kishikawa, ${ }^{1,2}$ Hiroshi Higuchi, ${ }^{1}$ Limei Wang, ${ }^{1}$ Nivedita Panch,, ${ }^{1}$ Valerie Maymi, ${ }^{3}$ Sachem Best, ${ }^{3}$ Samuel Lee, ${ }^{3}$ \\ Genso Notoya, ${ }^{2}$ Alex Toker, ${ }^{4}$ Lydia E. Matesic, ${ }^{5}$ Gerburg M. Wulf, ${ }^{6}$ Wenyi Wei, ${ }^{4}$ Motoyuki Otsuka, ${ }^{2}$ Kazuhiko Koike, ${ }^{2}$ \\ John C. Clohessy, ${ }^{1,3}$ Yu-Ru Lee, ${ }^{1,7}$ and Pier Paolo Pandolfi, ${ }^{1,8,9}$ \\ 'Cancer Research Institute, Beth Israel Deaconess Cancer Center, Department of Medicine, Beth Israel Deaconess Medical Center, Harvard Medical School, Boston, Massachusetts, USA. ${ }^{2}$ Department \\ of Gastroenterology, Graduate School of Medicine, The University of Tokyo, Tokyo, Japan. ${ }^{3}$ Preclinical Murine Pharmacogenetics Facility and Mouse Hospital, and ${ }^{4}$ Department of Pathology, Beth Israel \\ Deaconess Medical Center, Harvard Medical School, Boston, Massachusetts, USA. ${ }^{5}$ Department of Biological Sciences, University of South Carolina, Columbia, South Carolina, USA. ${ }^{6}$ Cancer Research Institute, \\ Beth Israel Deaconess Cancer Center, Department of Medicine, Division of Hematology/Oncology, Beth Israel Deaconess Medical Center, Harvard Medical School, Boston, Massachusetts, USA. IInstitute of \\ Biomedical Sciences, Academia Sinica, Taipei, Taiwan. ${ }^{8}$ Department of Molecular Biotechnology and Health Sciences, Molecular Biotechnology Center, University of Torino, Torino, Italy. ${ }^{9}$ Renown Institute for \\ Cancer, Nevada System of Higher Education, Reno, Nevada, USA.
}

\begin{abstract}
Activation of the phosphatidylinositol 3-kinase (PI3K) signaling pathway is a pervasive event in tumorigenesis due to PI3K mutation and dysfunction of phosphatase and tensin homolog deleted on chromosome 10 (PTEN). Pharmacological inhibition of PI3K has resulted in variable clinical outcomes, however, raising questions regarding the possible mechanisms of unresponsiveness and resistance to treatment. WWP1 is an oncogenic HECT-type ubiquitin E3 ligase frequently amplified and mutated in multiple cancers, as well as in the germ lines of patients predisposed to cancer, and was recently found to activate PI3K signaling through PTEN inactivation. Here, we demonstrate that PTEN dissociated from the plasma membrane upon treatment with PI3K inhibitors through WWP1 activation, whereas WWP1 genetic or pharmacological inhibition restored PTEN membrane localization, synergizing with PI3K inhibitors to suppress tumor growth both in vitro and in vivo. Furthermore, we demonstrate that WWP1 inhibition attenuated hyperglycemia and the consequent insulin feedback, which is a major tumor-promoting side effect of PI3K inhibitors. Mechanistically, we found that AMPK 22 was ubiquitinated and, in turn, inhibited in its activatory phosphorylation by WWP1, whereas WWP1 inhibition facilitated AMPK $\alpha 2$ activity in the muscle to compensate for the reduction in glucose uptake observed upon PI3K inhibition. Thus, our identification of the cellautonomous and systemic roles of WWP1 inhibition expands the therapeutic potential of PI3K inhibitors and reveals new avenues of combination cancer therapy.
\end{abstract}

\section{Introduction}

Phosphatidylinositol 3-kinases (PI3Ks) play a critical role in regulating various biological functions, including cell growth, survival, differentiation, metabolism, and motility. Dysregulation of the PI3K signaling pathway is one of the most frequently observed abnormalities in various types of cancer, including breast cancer, and leads to uncontrolled tumor cell growth and drug resistance $(1,2)$. Substantial efforts have therefore been devoted to developing agents targeting the PI3K/AKT/mTOR pathway, but these interventions have not been as effective as expected, mainly because of acquired resistance and ontarget toxicities such as hyperglycemia and immune deficiency $(3,4)$. On the other hand, negative regulators of this signaling

Conflict of interest: PPP and WW are cofounders of Rekindle Pharmaceuticals. The company is developing therapies for cancer.

Copyright: () 2021, American Society for Clinical Investigation.

Submitted: May 19, 2020; Accepted: October 27, 2021; Published: December 15, 2021

Reference information: J Clin Invest. 2021;131(24):e140436.

https://doi.org/10.1172/JCl140436. cascade also ensure timely inhibition of the PI3K pathway and thereby prevent excessive growth.

Phosphatase and tensin homolog deleted on chromosome 10 (PTEN), a dual-specificity lipid and protein phosphatase, is a critical upstream member of the cascade that efficiently dephosphorylates phosphatidylinositol trisphosphate (PIP3) and terminates propagation of the signal to the downstream AKT pathway $(5,6)$. PTEN partial loss of expression is frequently observed in various types of cancer, and, importantly, subsequent PTEN loss causes resistance to PI3K inhibitors, implying that PTEN plays an essential role in treatment with PI3K inhibitors (7-9).

PTEN dynamics at the plasma membrane has been recently shown to represent a critical on/off switch toward PI3K activation $(5,6)$. Specifically, PTEN ubiquitination by the proto-oncogenic WWP1, a HECT-type ubiquitin E3-ligase, was found to suppress PTEN localization at the plasma membrane and the ability of PTEN to counter PI3K activation (10). Given these facts, we focused on PTEN dynamics and activity upon treatment with PI3K inhibitors and, surprisingly, found that PTEN was dissociated from the plasma membrane and inactivated through the 
recruitment of WWP1 to the plasma membrane, and that membrane localization could be restored upon genetic and pharmacological inhibition of WWP1. Importantly, we further show that WWP1 inhibition attenuated hyperglycemia and subsequent hyperinsulinemia through the activation of AMPK, which offered a systemic advantage that countered the most common and tumor-promoting on-target toxicity of PI3K inhibition.

\section{Results}

While evaluating PTEN status upon PI3K inhibition, we discovered that this treatment markedly decreased the membrane fraction of PTEN in both triple-negative breast cancer (TNBC) and estrogen receptor-positive (ER-positive) breast cancer cells, although there was no prominent change in total PTEN levels (Figure 1, A and B). These effects on PTEN dissociation from the membrane were observed in cells treated with multiple PI3K inhibitors, including isoform-specific P110 inhibitors (Figure 1C and Supplemental Figure 1A; supplemental material available online with this article; https://doi.org/10.1172/JCI140436DS1). In line with our previous findings that PTEN localization is regulated by its ubiquitination status $(10,11)$, we found that, upon PI3K inhibition, PTEN dissociation from the plasma membrane was accompanied by increased ubiquitination (Figure 1D). As we recently reported that WWP1 was a critical regulator of PTEN membrane localization (10), we therefore evaluated whether PTEN subcellular localization upon treatment with a PI3K inhibitor would be affected when WWP1 was depleted. Indeed, depletion of WWP1 led to PTEN relocalization to the membrane in both MDA-MB-231 and MCF7 cells (Figure 2, A and B, and Supplemental Figure 1B) and reduced PTEN ubiquitination upon BKM120 treatment (Figure 2C), suggesting that WWP1 was responsible for both the increased PTEN ubiquitination and its subsequent dissociation from the membrane following BKM120 treatment.

To further explore the molecular mechanisms that lead to the activation of WWP1 upon PI3K inhibition, we examined the dynamics of intracellular localization of WWP1. We observed that both the membrane fraction of WWP1 (Figure 1, A and B) and its binding to PTEN increased after PI3K inhibitor treatment (Supplemental Figure 1C). Importantly, we also found that WWP1 bound to $\mathrm{PI}(4,5) \mathrm{P}_{2}$ more robustly than to $\mathrm{PI}(3,4,5) \mathrm{P}_{3}$ (Supplemental Figure 1D), suggesting that WWP1 was recruited and held to the plasma membrane through binding to PI $(4,5)$ $\mathrm{P}_{2}$ upon PI3K inhibition, which drastically increased the ratio of $\mathrm{PI}(4,5) \mathrm{P}_{2}$ to $\mathrm{PI}(3,4,5) \mathrm{P}_{3}(12)$.

We next tested whether the therapeutic effect of PI3K inhibition is enhanced by PTEN relocalization to the plasma membrane. To this end, we treated multiple breast cancer cell lines with BKM120 upon WWP1 silencing. Indeed, cells with intact or monoallelic PTEN expression proved more sensitive to the combination of BKM120 and WWP1 genetic inactivation, while cells with homozygous deletion or epigenetic inactivation of PTEN were found to be resistant to WWP1 inhibition (Figure 2D, Supplemental Figure 2A, and Supplemental Table 1). Consistently, add-back of PTEN restored the synergistic effect of WWP1 depletion in PTENnull MDA-MB-468 cells (Supplemental Figure 2, B and C). These data collectively suggest that WWP1 inhibition could enhance the efficacy of PI3K inhibition through PTEN reactivation.
Continuous treatment with PI3K inhibitors commonly results in acquired resistance, which represents one of the challenges in the clinical application of these inhibitors that most urgently demands a solution $(1,4,13)$. We thus generated isogenic cells from MDA-MB-231 and MCF7 cell lines, which are resistant to BKM120 treatment, to explore the underlying mechanisms of this resistance (Supplemental Figure 3, A and B). Intriguingly, we found that WWP1 expression in each resistant clone from different cell lines was upregulated proportionally to the increasing concentrations of BKM120 (Figure 3A and Supplemental Figure 3C), whereas, consistently, PTEN ubiquitination was found elevated in the resistant cells (Figure 3B). Amplification or epigenetic upregulation of the proto-oncogene $M Y C$ is frequently observed in breast cancer that has acquired resistance to PI3K inhibitors $(14,15)$, and we have previously reported that WWP1 overexpression can be driven by Myc during cancer development (10). We therefore evaluated the Myc status in the resistant cell lines and found that Myc expression levels were indeed increased in both resistant cell lines (Figure 3A). Additionally, we observed that Myc bound to the promoter region of the WWP1 gene more strongly in the resistant MDA-MB-231 high-dose (HD) cells than in the parental cell line (Supplemental Figure 3D), suggesting that activation of the Myc/ WWP1 pathway is one of the potential causes of resistance to PI3K inhibitors through PTEN dysfunction. To further corroborate the role of WWP1 in acquired resistance, we next generated MDAMB-231 and MCF7 cells, which stably overexpress WWP1. Consistently, WWP1 overexpression limited the effect of PI3K inhibitors in suppressing AKT and S6 activation, rendering cells resistant to the treatment (Figure 3, C-E), while the membrane localization of PTEN was reduced in WWP1-overexpressing MCF7 cells (Supplemental Figure 3E). Conversely, WWP1 depletion in resistant cells potentiated the sensitivity to BKM120 or BYL719 treatment (Figure 3, F and $\mathrm{G}$ ) and further inhibited the PI3K/AKT pathway (Figure $4 \mathrm{~A}$ and Supplemental Figure $3 \mathrm{~F}$ ).

We have previously shown that WWP1 is inhibited by indole-3-carbinol (I3C), a natural compound found in cruciferous plants (10). We thus tested the consequence of pharmacological WWP1 inhibition in mediating sensitivity to PI3K inhibitors in both parental and resistant cell lines. Indeed, pharmacological inactivation of WWP1 showed synergistic effects in combination with BKM120 or BYL719 in the suppression of cell growth (Figure 4, B and C, and Supplemental Figure 4, A and B). Notably, these effects were more prominent in resistant MDA-MB-231 cells with higher WWP1 expression (Figure 4, B and C), whereas only a negligible synergistic effect was observed in MDAMB-468 cells that displayed loss of PTEN (Supplemental Figure 4C). Together, these findings suggest a critical role for the WWP1/ PTEN pathway in mediating both sensitivity and acquired resistance to PI3K inhibitors.

It is broadly accepted that PI3K inhibitors induce acute insulin resistance, which results in severe hyperglycemia and subsequent hyperinsulinemia due to increased glucose release from the liver and suppressed glucose uptake in systemic tissues such as muscle and adipose tissues $(1,16)$. This on-target toxicity is widely perceived as a limit on the tolerance for and therapeutic efficacy of these compounds, as episodes of hyperglycemia have been experienced by $35.7 \%-63.7 \%$ of patients undergoing PI3K 
A
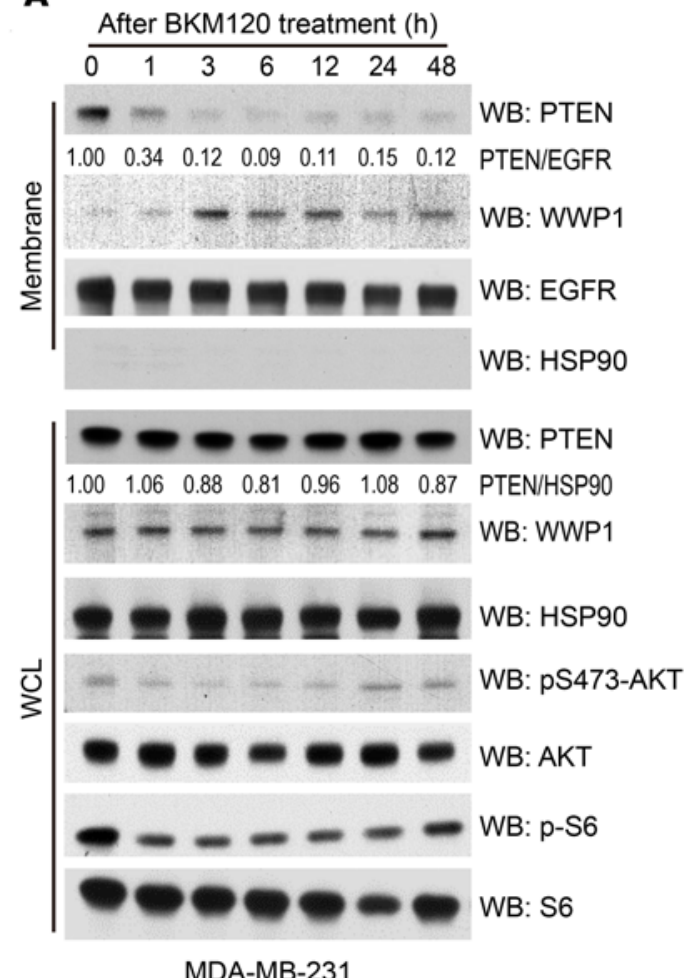

C

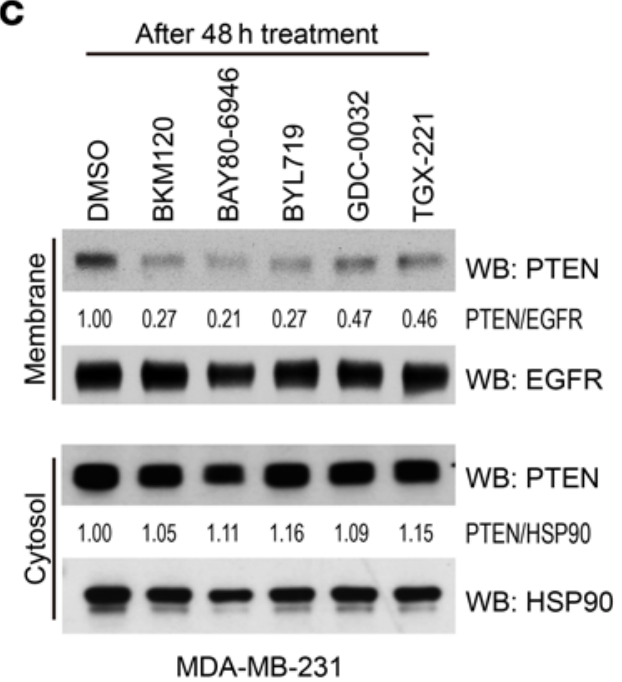

B
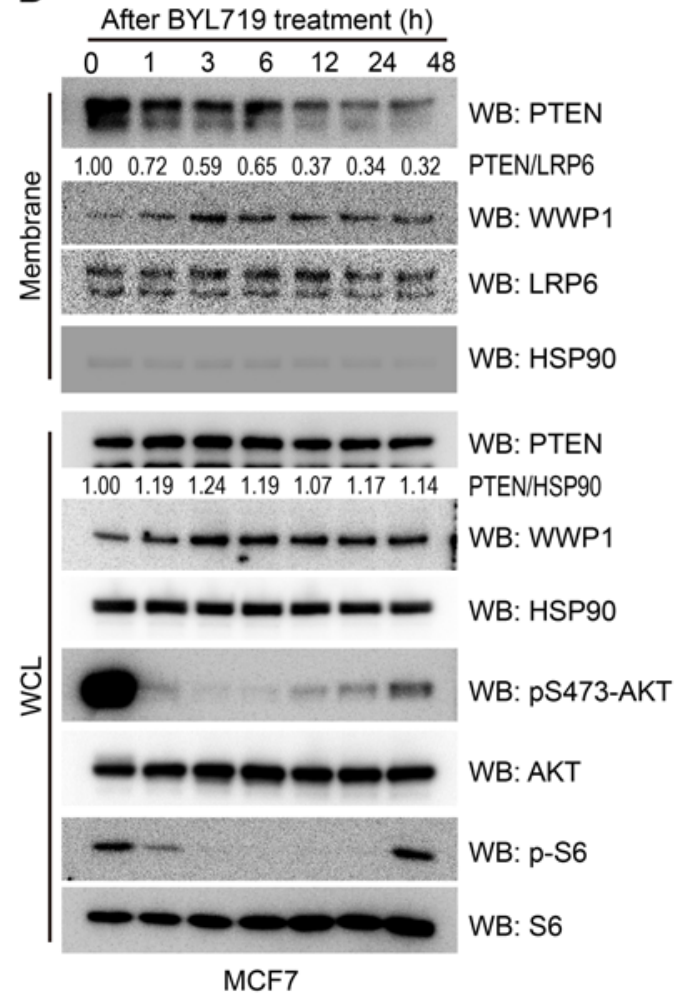

D

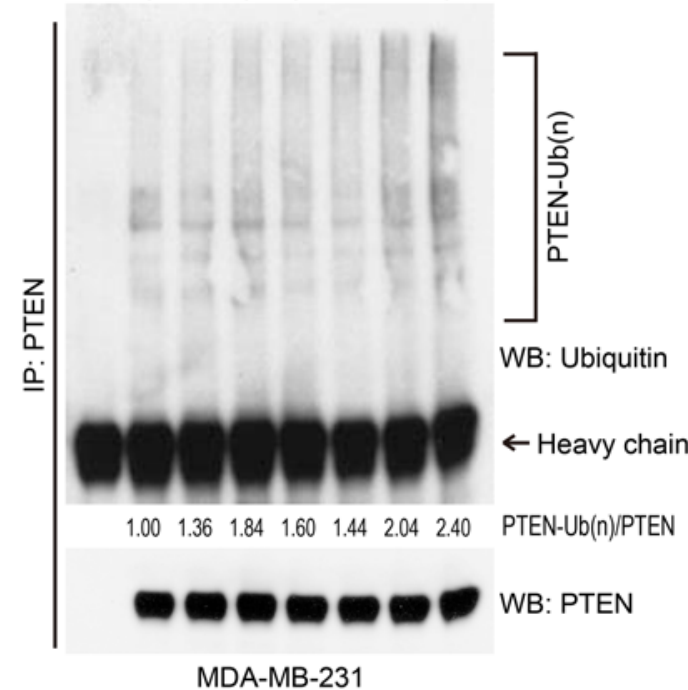

Figure 1. PTEN is ubiquitinated and dissociated from the plasma membrane upon treatment with PI3K inhibitors. (A) Evaluation of subcellular localization of PTEN upon BKM120 treatment. Membrane fractions and whole-cell lysate (WCL) were extracted from MDA-MB-231 cells treated with BKM120 over time and subsequently analyzed by WB. EGFR served as a membrane marker and HSP9O as the internal control. (B) Longitudinal subcellular localization of PTEN in MCF7 cells upon treatment with BYL719. LRP6 served as a membrane marker. Blots are from duplicate gels run in parallel. (C) Analysis of PTEN membrane fraction upon treatment with various PI3K inhibitors. MDA-MB-231 cells were treated with the indicated drugs for 48 hours, and membrane

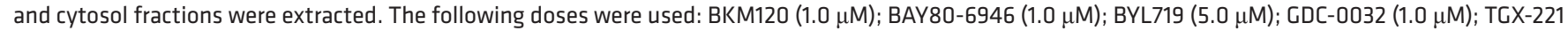
$(5.0 \mu \mathrm{M})$. The negative loading control for each fraction is shown in Supplemental Figure 1A as a technical replicate. (D) Analysis of endogenous PTEN ubiquitination over time after BKM120 treatment. Whole lysate was extracted from MDA-MB-231 cells treated with BKM120 for the indicated durations and immunoprecipitated with anti-PTEN beads. Arrow indicates the band with the mouse anti-lgG heavy-chain antibody. Numbers below the blot lanes represent the relative intensities of the PTEN band normalized to the respective loading control. PTEN-Ub(n)/PTEN, relative intensity of polyubiquitinated PTEN normalized to precipitated total PTEN. 
A
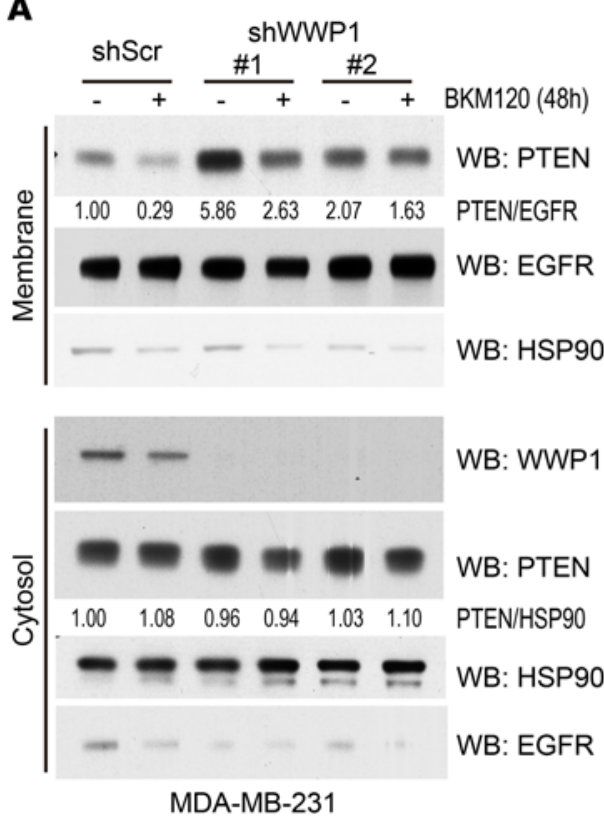

C

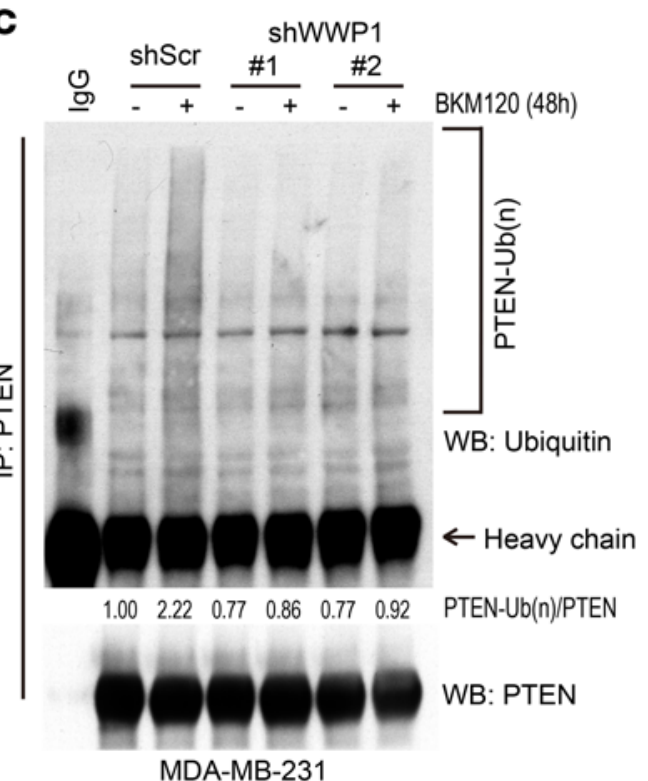

B

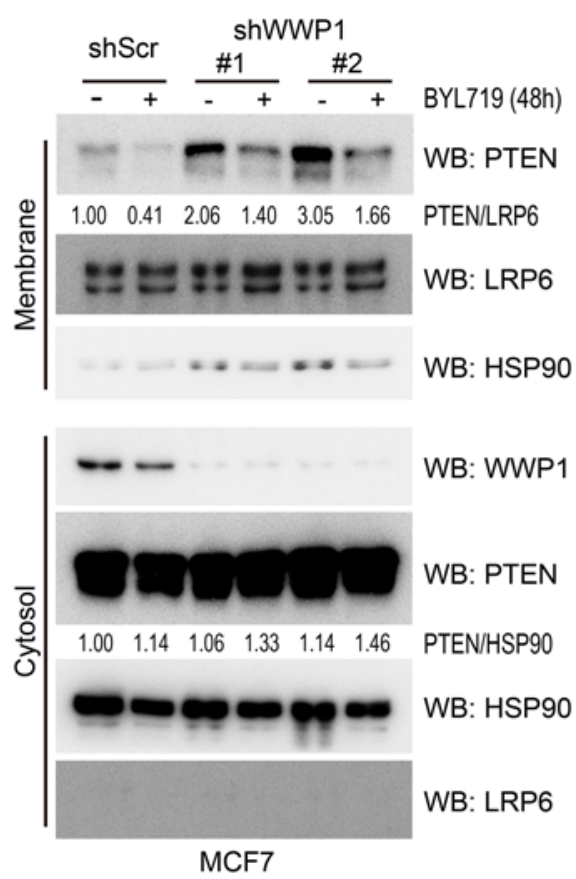

D

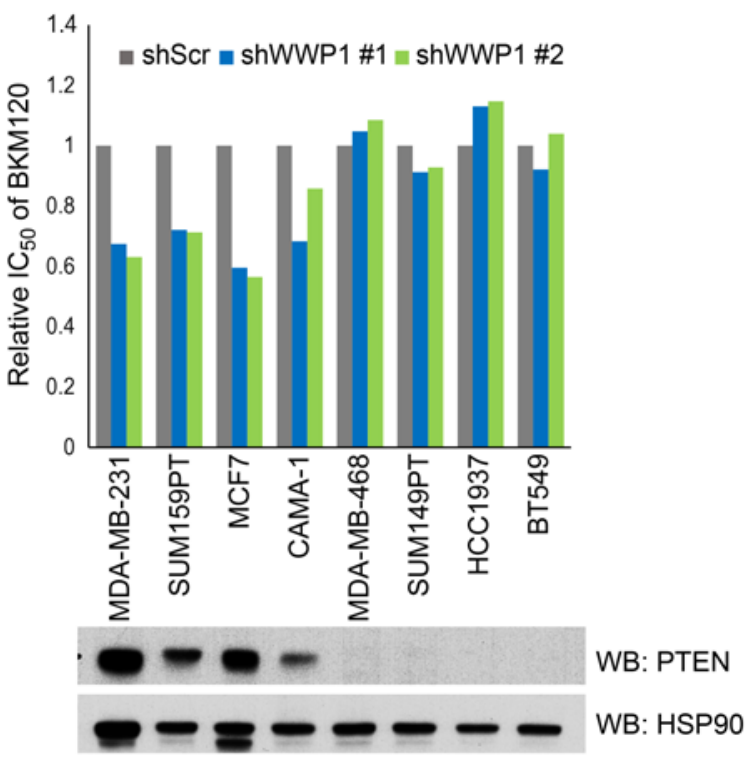

Figure 2. Membrane localization of PTEN is restored by WWP1 depletion, which increases the sensitivity of the PI3K inhibitor. (A) Membrane and cytosol fractions isolated from MDA-MB-231 cells stably expressing WWP1 shRNA (shWWP1) or scrambled (shScr). Cells were treated with DMSO or BKM120 for 48 hours. (B) Subcellular fraction of MCF7 cells stably expressing shWWP1 or shScr. Cells were treated with DMSO or $1 \mu$ M BYL719 for 48 hours. (C) Analysis of endogenous PTEN ubiquitination in MDA-MB-231 cells with WWP1 shRNA, which were treated with DMSO or BKM120 for 48 hours. (D) Evaluation of the $\mathrm{IC}_{50}$ of breast cancer cells with WWP1 depletion. The relative $\mathrm{IC}_{50}$ ratio was determined by dividing each $\mathrm{IC}_{50}$ value by that of the shScr. The PTEN expression status of each cell was evaluated by WB.

inhibitor treatment $(1,13,17-20)$. On this basis, we therefore tested whether targeting WWP1 would also affect blood glucose and insulin levels. First, we treated whole-body Wwp1-knockout mice $\left(W w p 1^{-/}\right)$and control mice $\left(W w p 1^{+/+}\right)$with BKM120 and monitored their blood glucose and insulin levels in vivo. Intriguingly, after BKM120 treatment, blood glucose levels in $\mathrm{Wwp}^{-1 /}$ mice fell to normal levels after an initial acute elevation, while sustained higher levels of glucose were observed in $\mathrm{Wwp}^{1^{+/+}}$mice (Figure
5A). We observed a similar attenuation of hyperinsulinemia followed by elevated blood glucose levels in $W w p^{1 /-}$ mice 3 hours after BKM120 treatment (Figure 5B). To further explore glucose dynamics upon pharmacological inactivation of WWP1 by I3C, we next monitored blood glucose levels in C57BL/6J mice pretreated for 1 week with I3C or metformin followed by BKM120 administration. I3C-pretreated mice showed significantly decreased glucose and insulin levels that were comparable to the decrease 
A

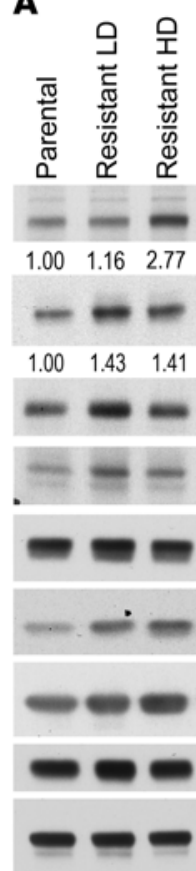

MDA-MB-231
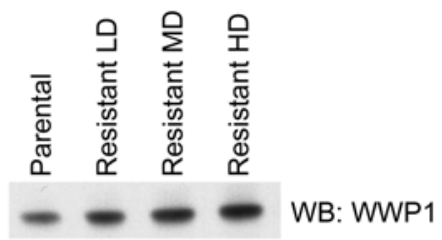

$\begin{array}{lllll}1.00 & 1.36 & 1.82 & 2.11 & \text { WWP1/HSP90 }\end{array}$

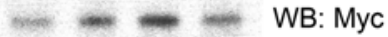

$\begin{array}{llll}1.00 & 1.46 & 1.79 & 1.67\end{array}$
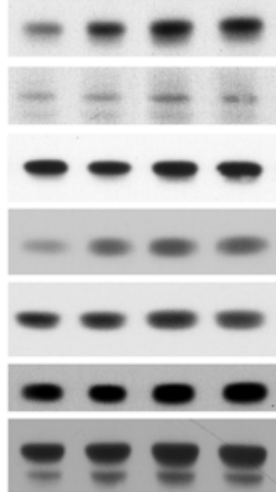

MCF7

WB: Myc

$\mathrm{Myc} / \mathrm{HSP} 90$

WB: AKT

WB: p-S6

WB: S6

WB: PTEN

WB: HSP90
WB: pS473-AKT

WB: pT308-AKT

C

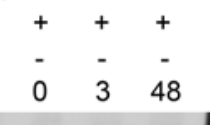

EV

HA-WWP1

BKM120 treatment $(\mathrm{h})$

WB: WWP1 (LE)

WB: WWP1 (SE)

WB: pS473-AKT

PAKT/AKT

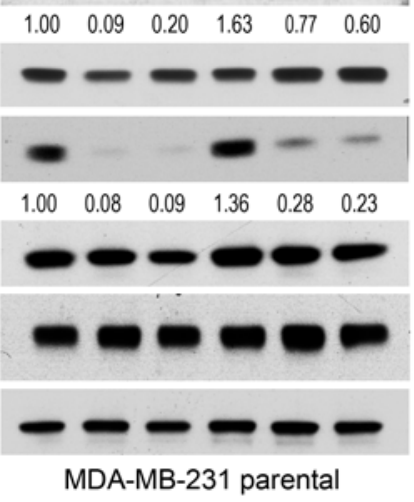

WB: AKT

WB: $\mathrm{p}-\mathrm{S} 6$

pS6/S6

WB: S6

WB: PTEN

WB: HSP9O

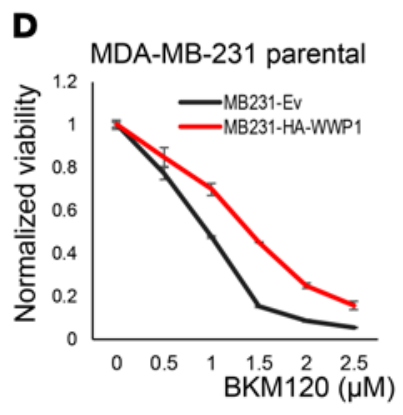

$\mathbf{F}$
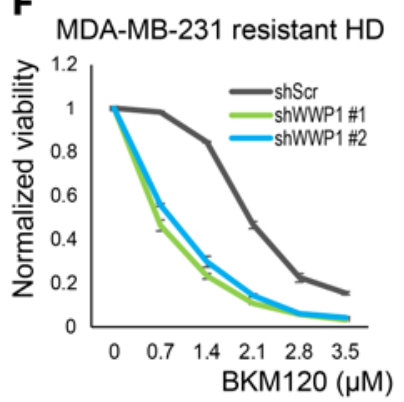

E

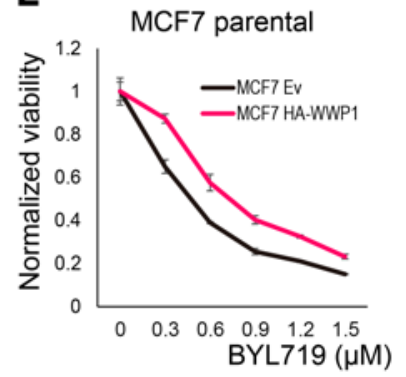

G

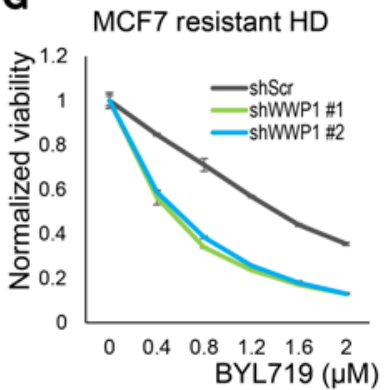

Figure 3. WWP1 expression is upregulated in isogenic breast cancer cells resistant to PI3K inhibitors. (A) Evaluation of WWP1 and Myc expression, and activity of the downstream PI3K pathway in isogenic resistant MDA-MB-231 and MCF7 cells. A set of isogenic resistant cells was sequentially established by repeating exposure to stepwise increasing concentrations of BKM120. Generated clones were maintained in BKM120-containing media. For the experiment, cells were cultured with BKM120-free media for 24 hours and harvested for WB. (B) Endogenous PTEN ubiquitination in isogenic MDA-MB-231 cells. Extracted lysates were pulled down by anti-PTEN antibody. (C) Activation of AKT and S6 in MDA-MB-231 cells with stable overexpression of WWP1 upon BKM120 treatment. Total lysates were resolved by SDS-PACE and subsequently probed with the indicated antibodies. EV, control empty vector. (D and E) Dose response curve of BKM120 in MDA-MB-231 (D) and BYL719 in MCF7 (E) cells with stable overexpression of HA-WWP1. (F and G) Dose response curve of BKM120 in resistant MDA-MB-231 HD cells (F) and BYL719 in resistant MCF7 HD cells (G) with WWP1 shRNA. Each dot indicates the mean \pm SD of a duplicated assay.

induced by metformin, which is extensively used to treat patients with diabetes (Figure 5, C and D) $(21,22)$.

AMPK is a known master regulator of glucose metabolism. It has been reported that WWP1 can degrade AMPK $\alpha$ (23) and that ubiquitination of AMPKa blocks its activation by competing for its phosphorylation (24). The catalytic $\alpha$ subunit of AMPK has 2 isoforms: $\alpha 1$ and $\alpha 2$. The AMPK $\alpha 2$ isoform is specifically highly expressed in heart and skeletal muscle, whereas AMPK $\alpha 1$ is 
A

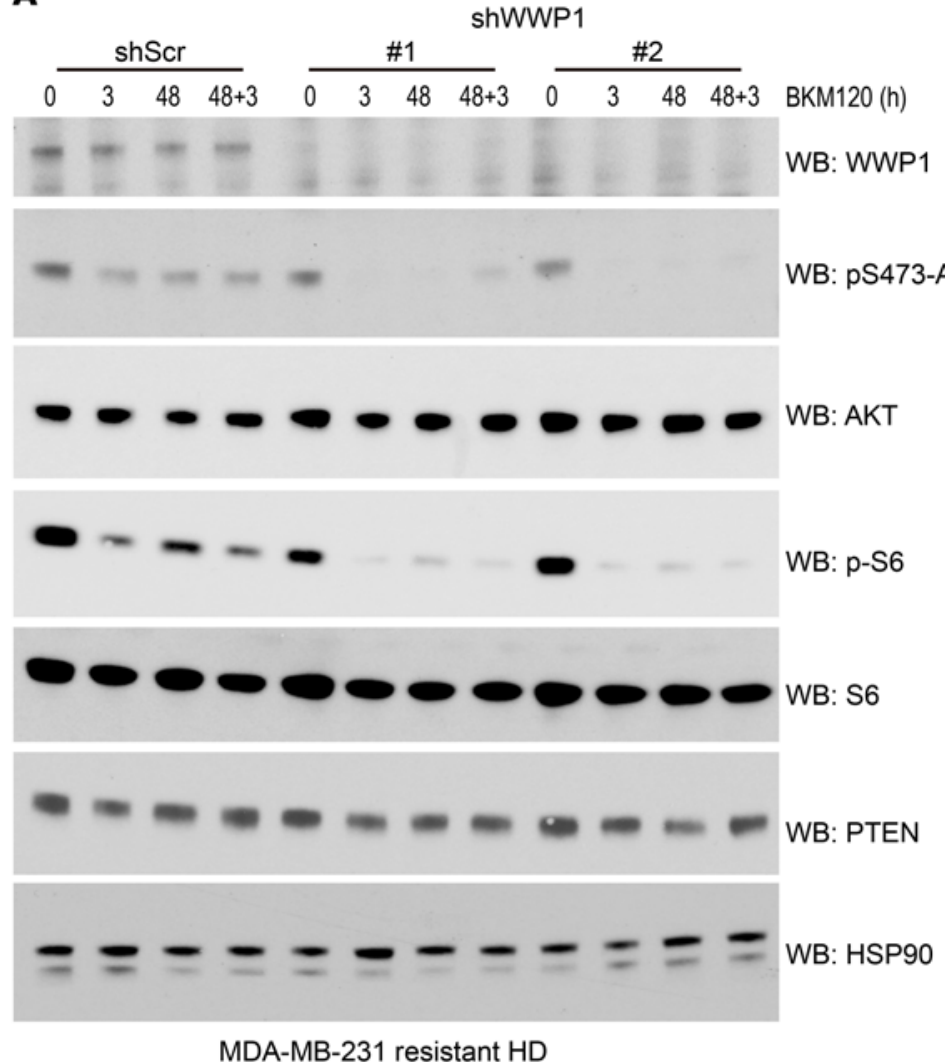

B

MDA-MB-231 parental

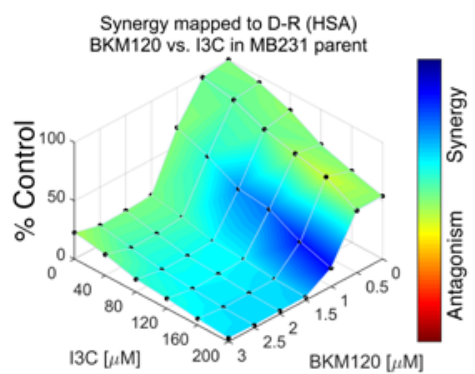

C

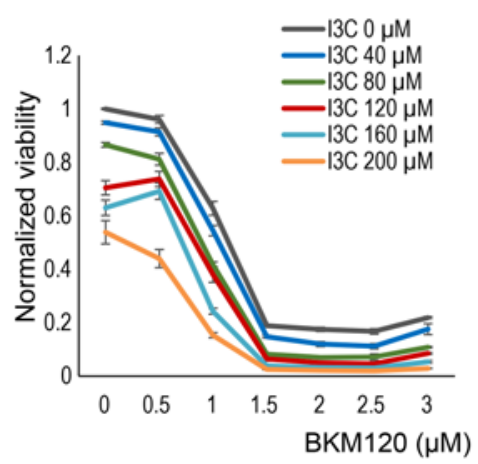

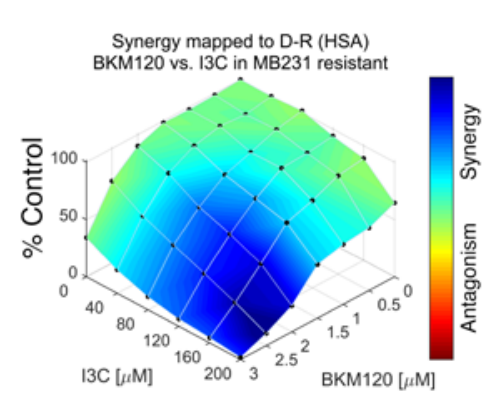

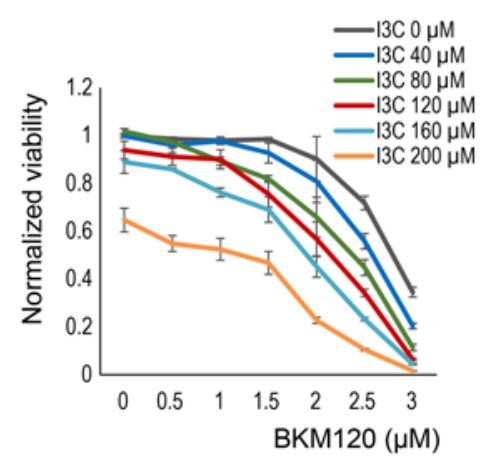

Figure 4. PI3K inhibitor efficacy is synergistically improved by WWP1 inhibition in resistant cells. (A) Analysis of AKT and S6 activity in resistant MDA-MB-231 HD cells stably expressing shWWP1. Cells were treated with BKM120 for the indicated durations and subjected to WB analysis. (B and C) $3 D$ plots of the synergy scores and dose response curves for combinatory treatment with I3C and BKM120 in MDA-MB-231 parental (B) and resistant $\mathrm{HD}$ (C) cells. Each dot shows the mean \pm SD of triplicate assays. Synergic performance scores were analyzed with Combenefit software. D-R, dose-response; HSA, highest single agent method.

ubiquitously expressed in whole tissues (Supplemental Figure $5 \mathrm{~A}$ ). On the other hand, we found that AMPK $\alpha 2$ was markedly suppressed in mouse breast tumor and human breast cancer cell lines (Supplemental Figure $5, \mathrm{~B}$ and $\mathrm{C}$ ), in accordance with previous reports that showed downregulation of AMPK $\alpha 2$ in primary breast cancer and hepatic cancer tissues (25-27). On the basis of these findings, we hypothesized that the substantial difference in expression profiles of the AMPK $\alpha$ isoform between breast cancer and skeletal muscle can trigger a differential response upon WWP1 and PI3K inhibition. We therefore examined whether WWP1 can systemically govern blood glucose and insulin levels through the regulation of AMPK functions in muscle. To investigate the functional interaction between WWP1 and each AMPK $\alpha$ isoform, we first performed in vivo ubiquitination assays in 293T cells expressing Myc-WWP1 and HA-AMPK. AMPK 2 2, but not AMP$\mathrm{K} \alpha 1$, was polyubiquitinated by WWP1 overexpression in a catalysis-dependent manner (Figure 6A). Consistent with a previous report demonstrating that activation of phosphorylation at Th172 of AMPK $\alpha$ is inhibited by its polyubiquitination (24), we observed that AMPK phosphorylation was suppressed in 293T cells upon overexpression of WWP1 (Supplemental Figure 5D). To further investigate AMPKa function upon double inhibition of PI3K and WWP1, we treated mouse C2C12 myoblasts with BKM12O in the setting of genetic or pharmacological inactivation of WWP1 and found that AMPK $\alpha$ was highly activated after BKM120 treatment in WWP1-inhibited cells, whereas AMPK $\alpha$ expression levels were relatively unaffected (Figure 6B and Supplemental Figure 5E). In contrast, we did not observe AMPK activation in MCF7 cells in which AMPKa1 was dominantly expressed (Supplemental Figure 5F). We further explored whether the adenosine diphosphate/ adenosine triphosphate (ADP/ATP) ratio could trigger phosphorylation of AMPK upon PI3K inhibition in C2C12 cells. The ADP/ATP ratio was slightly decreased in Wwp1-depleted cells but unaffected by the BKM120 treatment, suggesting that AMPK activation was induced by WWP1 and PI3K inhibition independently of ADP status (Supplemental Figure 5G). To further validate these in vitro findings, we next treated $W w p 1^{+/+}$ and $\mathrm{Wwp}^{1--}$ mice with BKM12O in vivo and found that AMPK was much more active in the muscle tissue of $W w 1^{-/-}$mice than in that of $W_{w p 1^{+/+}}$mice upon BKM12O treatment (Figure 6C and Supplemental Figure 6A).

AMPK is known to play crucial roles in glucose uptake through translocation to the cell surface of glucose transporter type 4 (GLUT4), which is mainly expressed in skeletal muscle and adipose tissues (28, 29). We therefore evaluated GLUT4 transport as a downstream effect of AMPK activity by quantitating surface GLUT4 levels in C2C12 cells stably expressing the Myc-GLUT4-mCherry fusion protein, in which a Myc tag is inserted into the N-terminus and exposed 
A
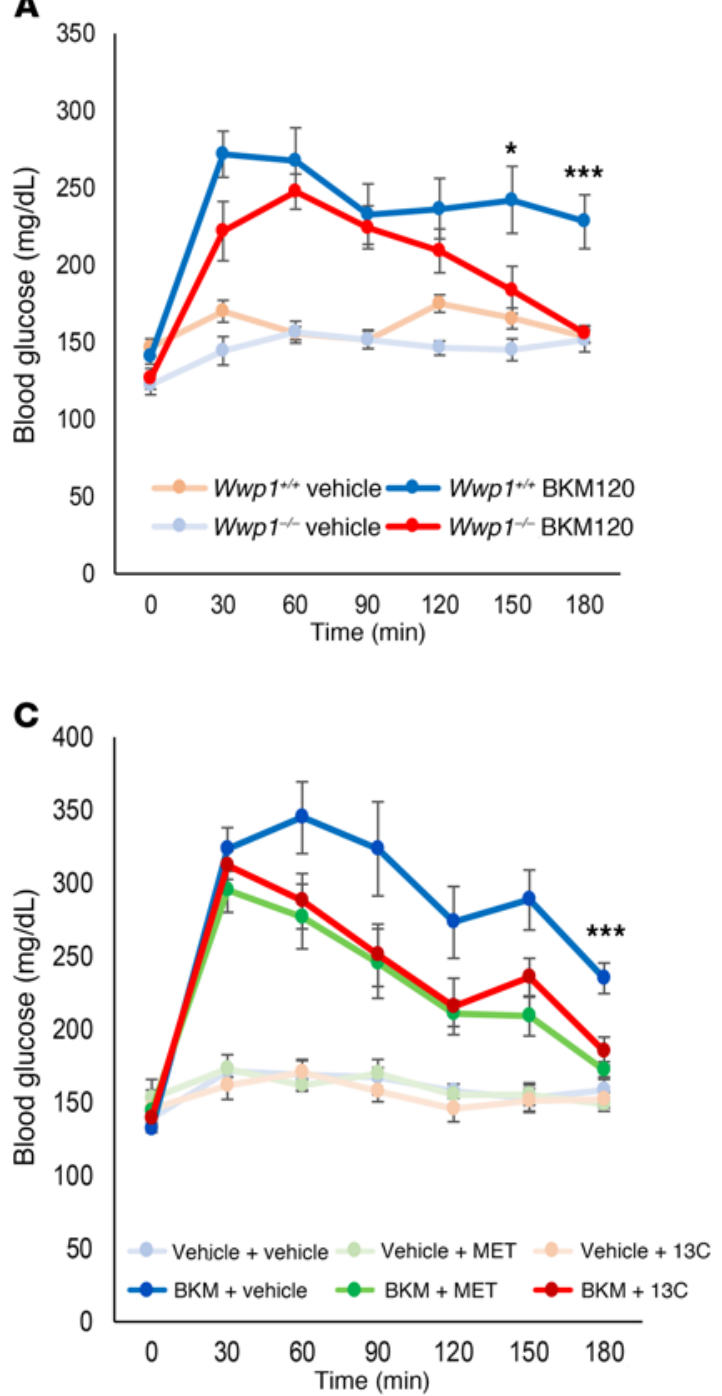

B

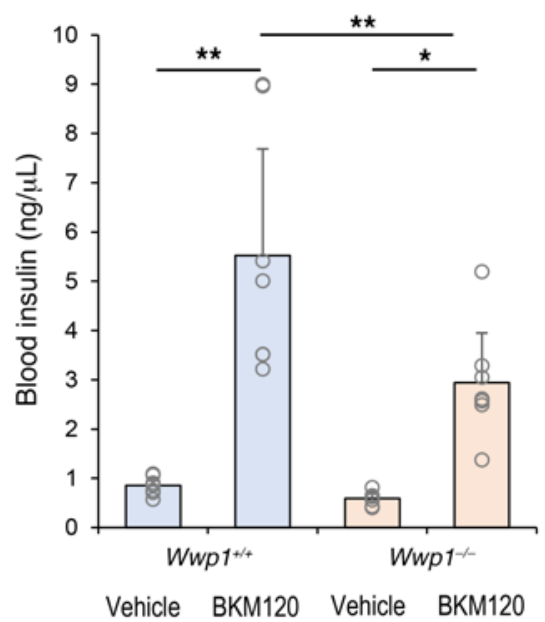

D

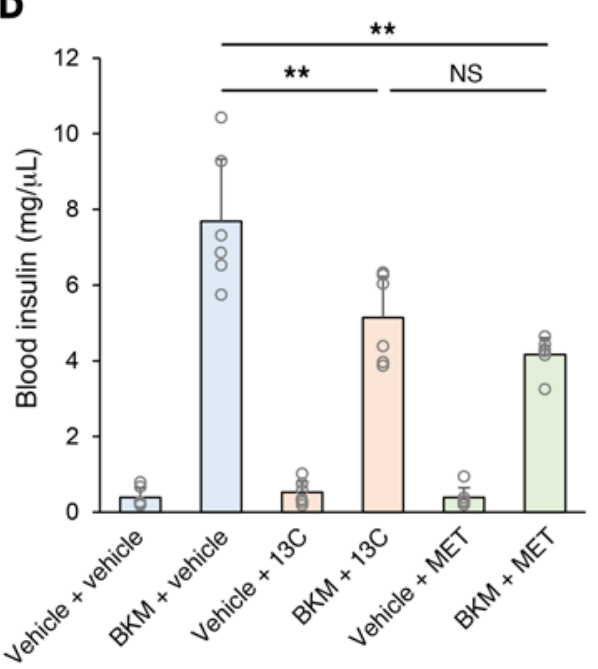

Figure 5. Hyperglycemia induced by PI3K inhibitor treatment is attenuated by the inhibition of WWP1. (A) Mean \pm SD of blood glucose levels in mice treated with vehicle or BKM120 (50 mg/kg, $n=9$ per group). (B) Mean \pm SD of blood insulin levels assessed 3 hours after BKM120 administration ( $n=7$ per group). (C) Mean \pm SD of blood glucose levels in mice after treatment with vehicle or BKM120 $(50 \mathrm{mg} / \mathrm{kg})$. Mice $(n=6$ per group) were pretreated with vehicle, I3C (50 mg/kg), or metformin (MET, $200 \mathrm{mg} / \mathrm{kg}$ ) for 7 days. (D) Mean \pm SD of blood insulin levels assessed 3 hours after BKM120 treatment $(n=6$ per group). ${ }^{*} P<0.05,{ }^{*} P<0.01$, and ${ }^{* *} P<0.005$, by repeated-measures 2-way ANOVA followed by Tukey-Kramer multiple-comparison test (A and $\mathbf{C}$ ) and 1-way ANOVA followed by Tukey-Kramer multiple-comparison test (B and $\mathbf{D})$.

outside the cell membrane, while mCherry is fused at the internal C-terminus (30). In line with our other findings, the reduction in the surface GLUT4 ratio after BKM120 treatment was rescued in Wwp1-depleted cells (Figure 6D and Supplemental Figure 6B). Similarly, glucose uptake was restored after BKM120 treatment in Wwp1-depleted cells, an effect that was antagonized by depletion of Ampk. By contrast, Pten depletion did not affect glucose uptake in C2C12 shWWP1 cells (Figure 6E). Finally, to validate the impact of AMPK activation on glucose homeostasis in vivo, we treated $W w p 1^{1^{++}}$and $W w p 1^{-/-}$mice with BKM120 and the AMPK inhibitor compound C (CC). Consistent with the in vitro results, blood glucose levels in $\mathrm{Wwp}^{-1-}$ mice were elevated by CC treatment upon PI3K inhibition (Supplemental Figure 6C). Collectively, these data suggest that the impairment in the glucose uptake cascade triggered by PI3K/AKT inhibition can be restored, at least in a part, by further activation of AMPK elicited by WWP1 inhibition in muscle tissues. In contrast, we found that glucose uptake was not rescued in WWP1-depleted MDA-MB-231 cells under BKM120 treatment (Supplemental Figure 6D), suggesting that the impact of AMPK activation on glucose uptake was not sufficient to counter the profound consequences of combined PI3K inhibition and PTEN reactivation in conditions in which AMPK $\alpha 2$ levels were significantly reduced.

On the basis of the results showing that WWP1 inhibition combined with PI3K inhibitor treatment was beneficial both systemically and in tumors, we next evaluated the consequence of combined pharmacological targeting of PI3K and WWP1 for cancer treatment in 3D culture assays as well as in vivo. To this end, we first validated the synergistic effects of targeting WWP1 along with multiple PI3K inhibitors in soft agar colony formation 
A
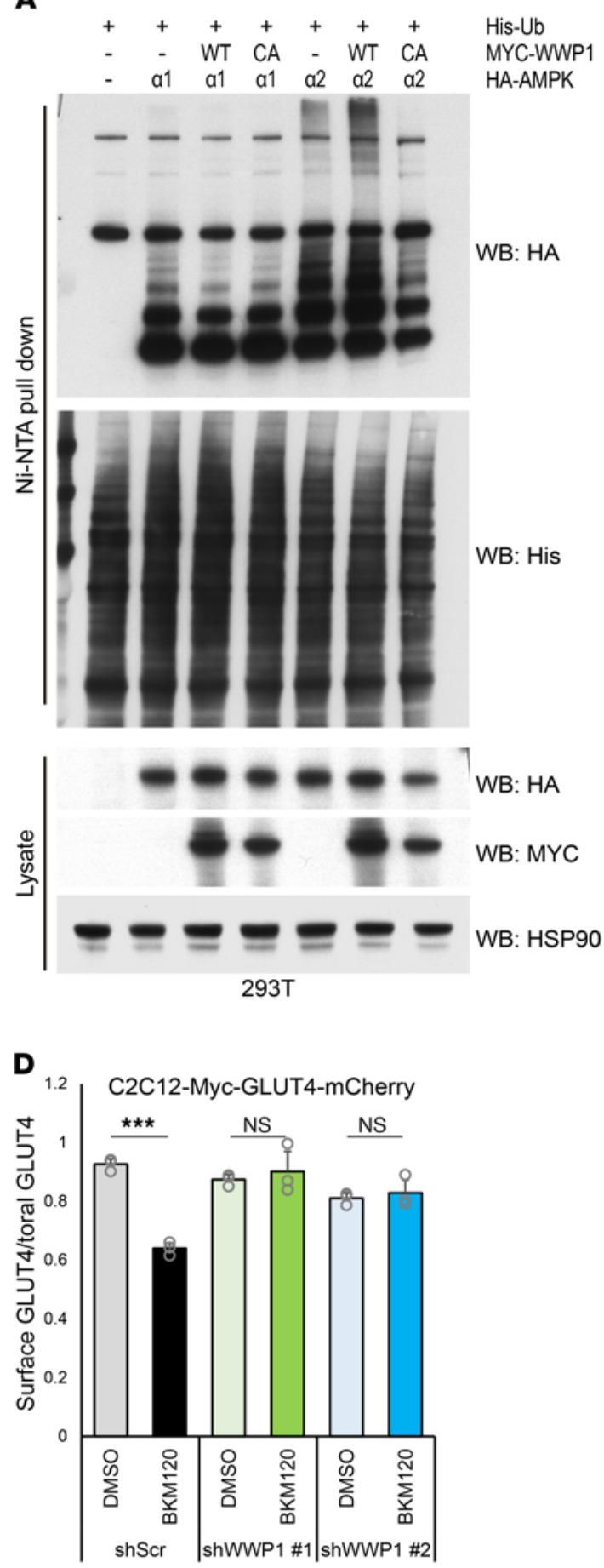

B

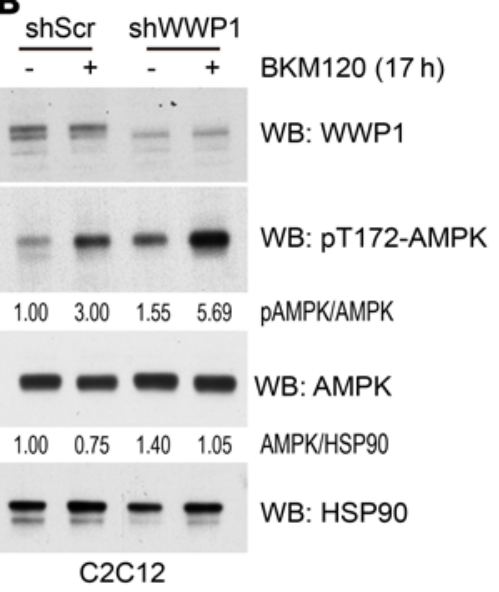

C

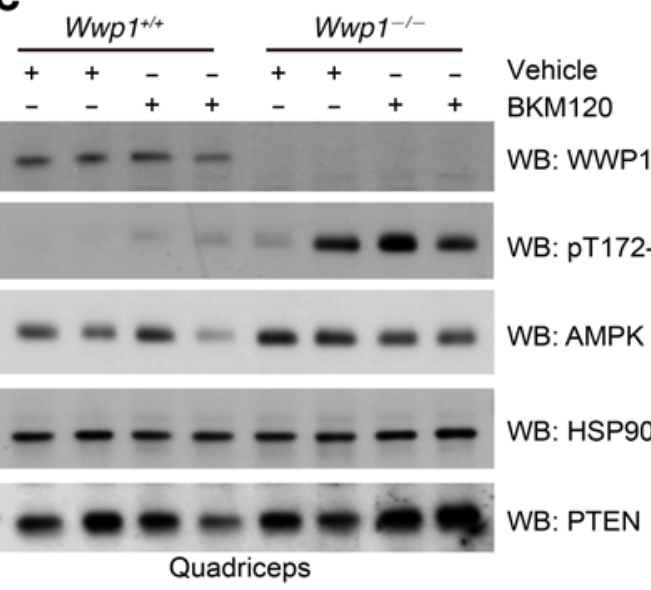

E

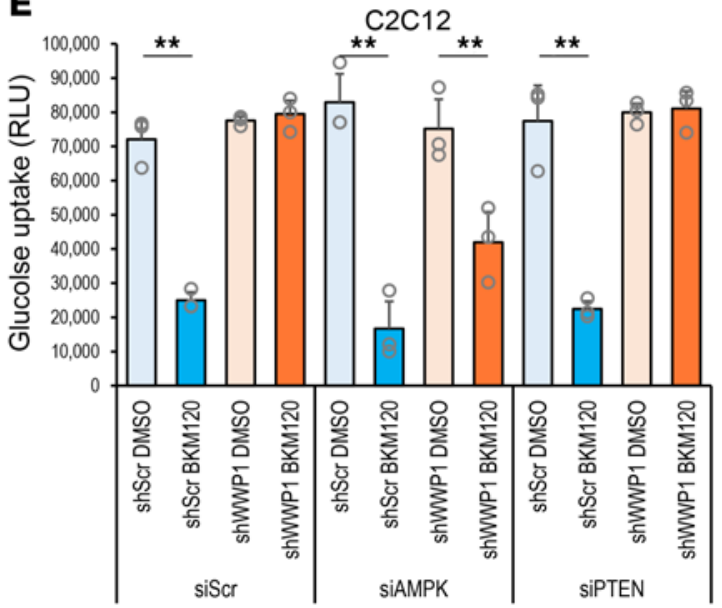

Figure 6. Glucose uptake is restored by WWP1 inhibition through the activation of AMPK $\alpha 2$. (A) Analysis of polyubiquitination of the AMPK $\alpha$ isoform in 293T cells. CA, catalytically inactive mutant. (B) Assessment of AMPK activity in Wwp1-depleted C2C12 cells. Differentiated C2C12 cells were treated with BKM120 $(2.0 \mu \mathrm{M})$ for 17 hours with serum starvation and subjected to WB analysis. Blots are from duplicate gels run in parallel. (C) Evaluation of AMPK activation in quadriceps muscle tissue from $W w p 1^{+/+}$and $W w p 1^{-/-}$mice. Mice starved for 3 hours were treated with vehicle or BKM120 (50 $\mathrm{mg} / \mathrm{kg}$ ) for 1 hour. Data shown are representative of 2 independent experiments. (D) Quantification of GLUT4 localized on the cell surface upon BKM120 treatment. C2C12-Myc-GLUT4-mCherry cells with stable expression of WWP1 shRNA were treated with DMSO or BKM120 for 17 hours and subjected to immunofluorescence staining with anti-Myc-tagged antibody, followed by measurement via flow cytometry. Each plot shows the mean \pm SD of triplicate assays. (E) Quantitation of glucose uptake upon BKM120 treatment. C2C12-shWWP1 cells transduced with AMPK or PTEN siRNA were starved and treated with BKM120 for 2 hours followed by insulin stimulation for 15 minutes. Each plot shows the mean \pm SD of triplicate assays. ${ }^{* *} P<0.01$ and ${ }^{* * *} P<0.005$, by 2-tailed $t$ test (D) and 1-way ANOVA followed by Tukey-Kramer multiple-comparison test (E). 
A
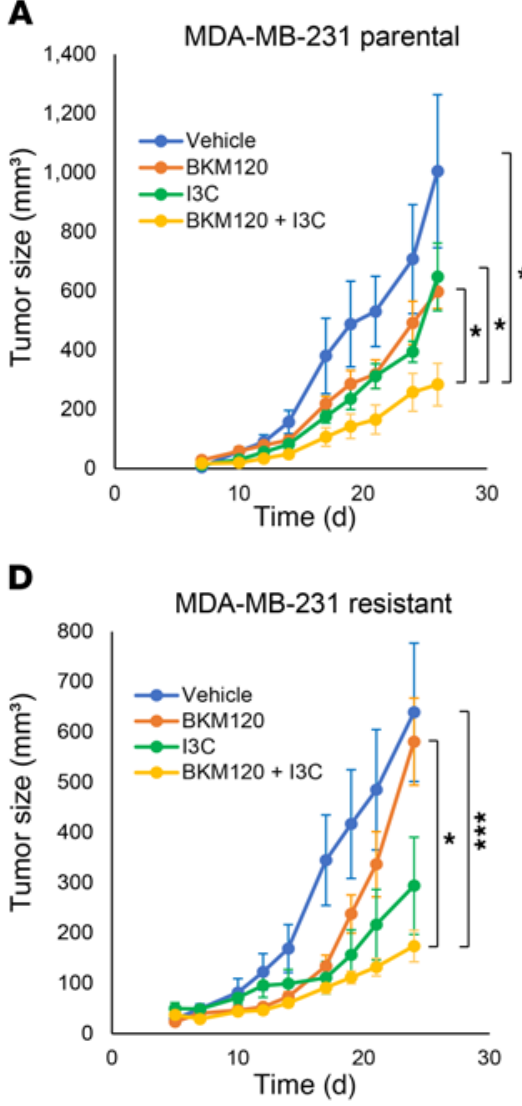

B

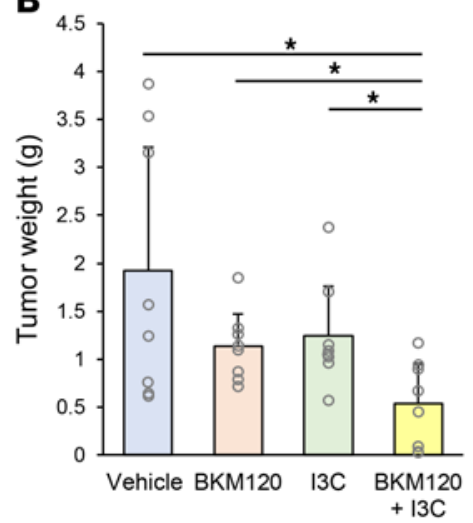

$\mathbf{E}$

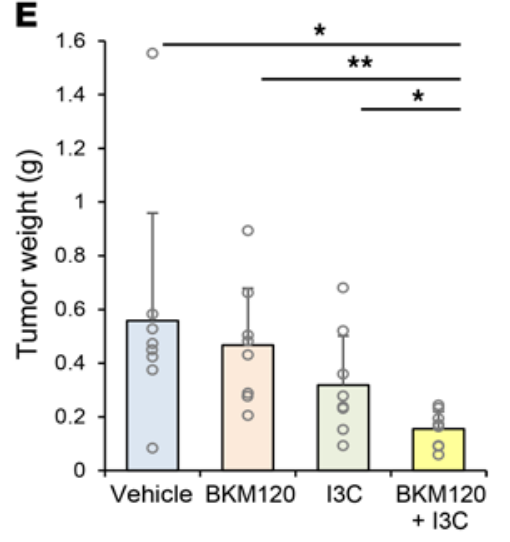

C

MDA-MB-231 parental

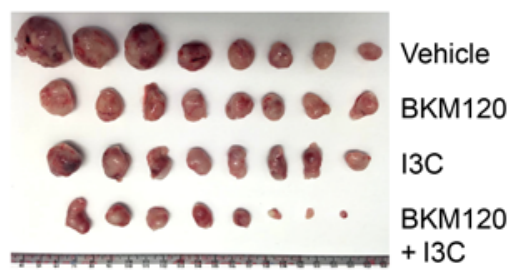

$\mathbf{F}$

MDA-MB-231 resistant

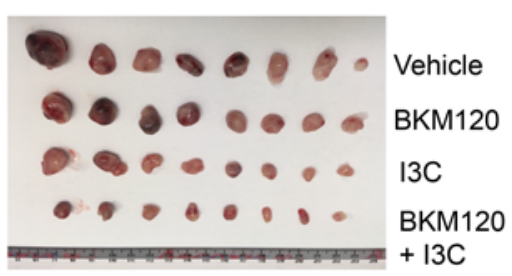

Figure 7. Combinatory therapy with I3C improves the efficacy of PI3K inhibitors. (A-C) Tumor xenograft assays were performed with subcutaneous implantation of MDA-MB-231 parental cells. Mice were treated orally with vehicle, BKM120 (30 mg/kg), $13 C(100 \mathrm{mg} / \mathrm{kg})$, or a combination of the drugs every day starting on day 3 after implantation. Data are shown as the mean \pm SEM for size and the mean \pm SD for weight $(n=8$ per group). (D-F) Analysis of the size and weight of xenografts of resistant MDA-MB-231 HD cells. Mice were treated with the indicated drugs at the same dose as that used in the parental cells starting on day 3 after implantation ( $n=8$ per group). Data are shown as the mean \pm SEM for size and mean \pm SD for weight. ${ }^{*} P<0.05$, ${ }^{*} P$ $<0.01$, and ${ }^{* *} P<0.005$, by linear mixed-effects model (A and $\mathbf{D}$ ) and 1-way ANOVA followed by Tukey-Kramer multiple-comparison test (B and $\left.\mathbf{E}\right)$.

assays using MDA-MB-231 cells. Indeed, we observed a significant decrease in colony numbers after treatment with PI3K inhibitors in WWP1-depleted cells (Supplemental Figure 7A). We next performed soft agar colony formation assays using MDA-MB-231 parental and PI3K inhibitor-resistant cells treated with a combination of BKM120 and I3C. We observed significant suppression after combinatorial treatment in both parental and resistant cells (Supplemental Figure 7, B and C). Importantly, we observed a profound tumor-suppressive effect in xenograft tumors treated with combined BKM120 and I3C in both parental and resistant TNBC cell lines, while the effect of BKM120 monotherapy was limited in resistant cells (Figure 7, A-F, and Supplemental Figure 7, D and E). This additive effect was also observed when we used BYL719 (alpelisib), a clinically approved PI3K inhibitor, in combination with I3C in ER-positive MCF7 xenografts (Supplemental Figure 8, $\mathrm{A}$ and B). Importantly, we also tested the effects of combinatorial treatments in patient-derived xenograft (PDX) models representative of ER-positive and TNBC breast cancers. In both models, we found that combination treatment synergistically and potently suppressed tumor growth compared with single-arm treatments (Figure 8, A-F), offering a new therapeutic opportunity for both PI3K inhibitor-sensitive and -resistant breast tumors (17).
In line with our previous findings, the elevation in blood insulin levels was also suppressed in the combination treatment arms of our preclinical trials in both cell line xenograft and PDX models (Supplemental Figure 8, C-E).

\section{Discussion}

Our findings reveal the existence of an important and, to our knowledge, previously unrecognized negative feedback mechanism that physiologically restrains PTEN function upon PI3K inactivation through WWP1. Genetic alternations that activate the $\mathrm{PI} 3 \mathrm{~K} / \mathrm{PTEN} / \mathrm{AKT} / \mathrm{mTOR}$ pathway are detected in $60 \%$ of breast cancers. In particular, PIK3CA is highly mutated in luminal-type breast cancer with positive ER expression. Accumulating evidence shows that PI3K inhibitors are more effective against PIK3CAmutant cancers, which show dependence for survival and growth on a dysregulated PI3K/AKT pathway (17). This explains why the indication of BYL719 (alpelisib), the first PI3K inhibitor to be approved in solid tumors, is for now limited to ER-positive and PIK3CA-mutant breast cancers. By contrast, patients with TNBC, even when harboring PIK3CA mutations, to date do not benefit from PI3K inhibitors or their combination with other chemotherapies $(20,31)$. In this study, we show that WWP1 inhibition 
A

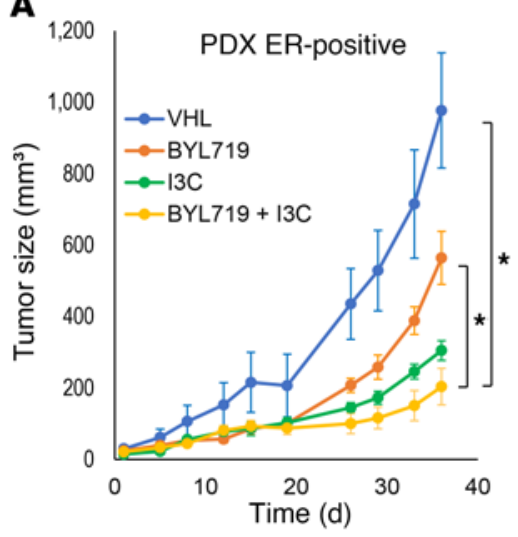

D

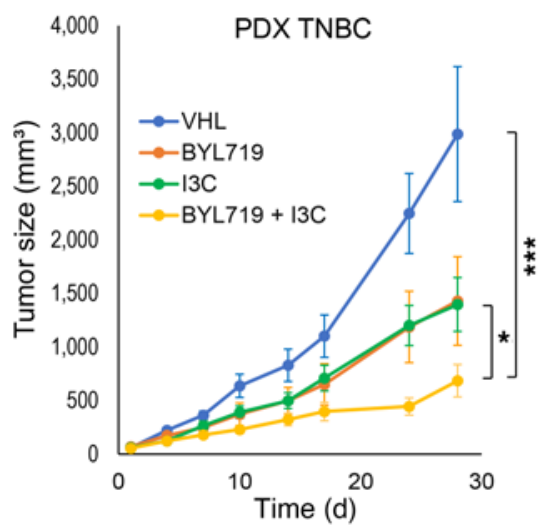

B

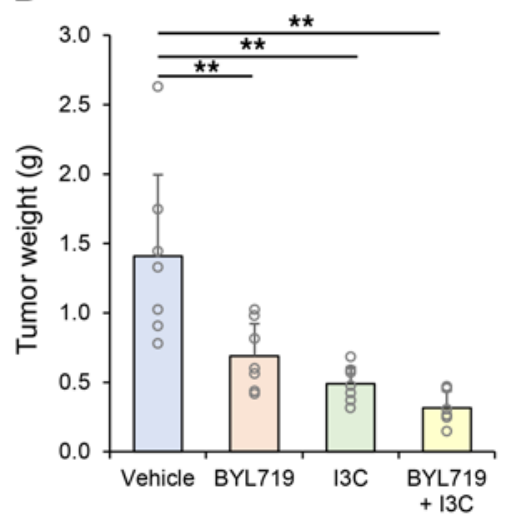

E

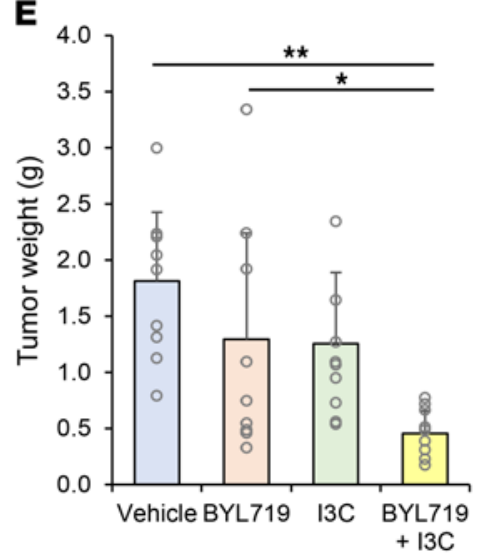

C

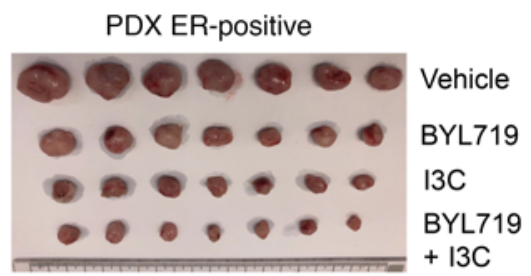

Figure 8. Double inhibition of PI3K and WWP1 suppresses tumor growth in PDX models. (A-C) Analysis of the size and weight of PDX tumors derived from ER-positive breast cancer ( $\mathrm{HCl}-011)$. Mice were treated orally with vehicle, BYL719 (20 mg/kg), I3C (200 mg/kg), or a combination of BYL719 and I3C, 5 days a week from week 4 after implantation ( $n=6$ in combo arm and 7 in the other arms). Data are shown as the mean \pm SEM for size and mean \pm SD for weight. (D-F) Analysis of the size and weight of the PDX derived from TNBC (TM00089). Mice were treated with the indicated compounds at the same dose as that used for the ER-positive PDX, starting on week 3 after implantation ( $n=10$ per group). Data are shown as the mean \pm SEM for size and mean \pm SD for weight. ${ }^{*} P<.05$, ${ }^{* *} P<0.01$, and ${ }^{* *} P<0.005$, by linear mixed-effects model (A and $\left.\mathbf{D}\right)$ and 1-way ANOVA followed by Tukey-Kramer multiple-comparison test (B and E).

enhanced the efficacy of PI3K inhibitors in both ER-positive and TNBC tumors, indicating that this combinatorial treatment can broaden the indication for use of PI3K inhibitors to different breast cancer subtypes including those that display acquired resistance to PI3K inhibitors. Indeed, WWP1 inhibition further deactivated the $\mathrm{PI} 3 \mathrm{~K} / \mathrm{AKT} / \mathrm{mTOR}$ pathway through the reactivation of PTEN, and this can be relevant in TNBC, where loss or suppression of PTEN is frequently observed, which in turn represents one possible reason for TNBC insensitivity to PI3K inhibitors $(9,32)$.

We found that WWP1 expression was upregulated in breast cancer cells resistant to a PI3K inhibitor, which paralleled Myc upregulation. We previously reported that deregulation of Myc expression directly promotes the WWP1/PTEN inhibitory axis, which activates the PI3K/AKT pathway (10). Collectively, these data suggest that elevation of WWP1 expression, which impairs the PI3K/AKT pathway-inhibitory role of PTEN, may often be driven by Myc upregulation, one of the most frequent events correlating with the acquisition of resistance to PI3K inhibitors (14, 15). Here, we found that overexpression of WWP1 had a relatively small impact on the sensitivity of PI3K inhibitors as compared with depletion of WWP1 (Figure 3, D and E). We hypothesize that endogenous WWP1 is already maximally activated toward PTEN function in the cells and that ectopic expression of WWP1 therefore does not greatly affect PTEN function or PI3K activity. In contrast, the depletion of endogenous WWP1 had a much more robust and impactful effect on the $\mathrm{IC}_{50}$ in these cells (Figure 3, F and G). In addition to WWP1 upregulation, Myc upregulation in resistant cells may also function to regulate genes and pathways that lead to resistance, which can further contribute to resistance in these cells. This may be another reason why increased WWP1 expression did not correlate with a substantial increase in the $\mathrm{IC}_{50}$ in isotopic resistant cells. However, from a therapeutic perspective, WWP1 can be a potent target to counter resistance to PI3K inhibition.

We further demonstrated that WWP1 inhibition was of clinical relevance, as it resulted in both cell-autonomous and systemic beneficial effects, particularly in the setting of PI3K inhibition. Genetic and pharmacological inactivation of WWP1 in tumor cells reactivated PTEN and further blocked the downstream PI3K pathway. Simultaneously, in muscle tissues, WWP1 inhibition activated the AMPK pathway and countered the systemic reduction of glucose uptake caused by PI3K inhibition, which in turn attenuated hyperglycemia and subsequent hyperinsulinemia (Figure 


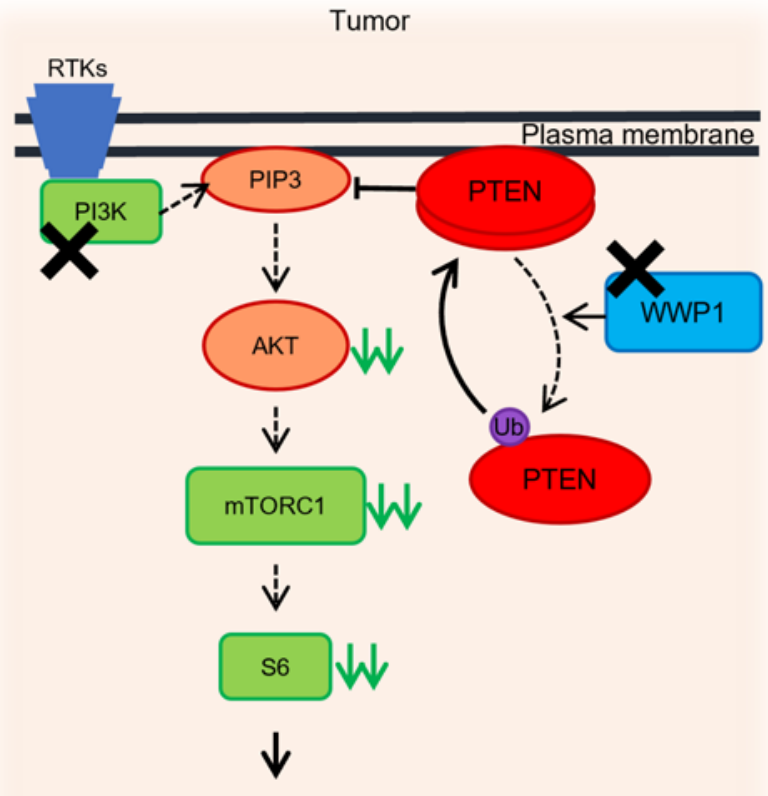

Restoration of PTEN function upon WWP1 inhibition overcomes resistance to PI3K inhibitors

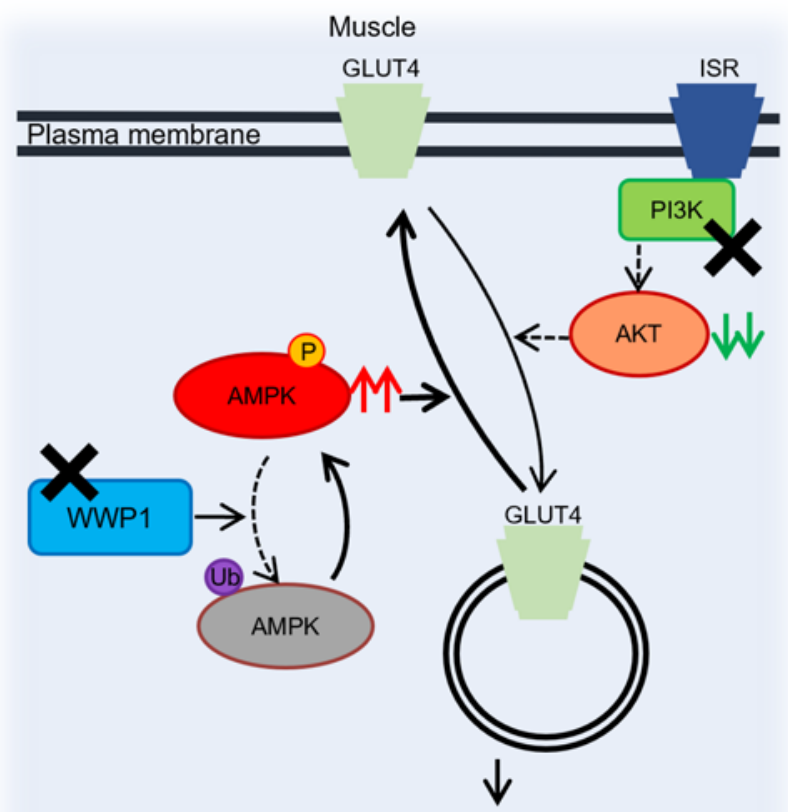

Glucose uptake restoration upon WWP1 inhibition

Figure 9. Cell-autonomous and systemic roles of WWP1 inactivation upon PI3K inhibition. Schematic illustration of the proposed model showing the cell-autonomous and systemic effects of inactivated WWP1 upon PI3K inhibition. PI3K inhibitors suppress the activity of AKT and the downstream signaling pathway, whereas PTEN is ubiquitinated and dissociated from the membrane through membrane recruitment of WWP1 in tumor tissues. WWP1 inhibition, by contrast, reactivates PTEN and further suppresses the PI3K/AKT pathway. In muscle tissues, GLUT4 is internalized into the cytoplasm, and glucose uptake is impaired upon PI3K inhibitor treatment, which causes systemic drug-induced hyperglycemia. The inactivation of WWP1 facilitates the phosphorylation of AMPK $\alpha 2$ and subsequently leads GLUT4 back onto the cell surface. RTKs, receptor tyrosine kinases; Ub, ubiquitin; ISR, insulin receptor; P, phosphorylation.

9). These cell-autonomous and systemic functions are extremely important from a clinical perspective, as both compensate for the intrinsic drawbacks of PI3K inhibitors: the insufficient inhibition of the downstream PI3K/AKT pathway due to the self-restraint of PTEN function and the elevation of systemic blood glucose and insulin levels. Furthermore, constitutive high blood glucose and insulin levels facilitate tumor growth through an insulin/ PI3K/mTOR feedback loop, which may also compromise the treatment effectiveness of PI3K inhibitors (1, 33). Importantly, I3C is a natural compound derived from cruciferous vegetables with negligible toxicity $(34,35)$, and thus this combinatorial treatment is easily applicable without increasing the risk of toxicity and so can play a significant role in enhancing the therapeutic effects of PI3K inhibitors in a wide range of patients with cancer who are resistant or intolerant to these drugs.

Moreover, double inhibition of PI3K and WWP1 can be expanded to other nonmalignant or premalignant syndromes caused by dysregulation of the PI3K/AKT pathway. For instance, it has recently been shown that treatment with low doses of BYL719 can yield dramatic therapeutic benefits for patients with PIK3CA-related overgrowth spectrum (PROS), which is caused by postzygotic mosaic gain-of-function mutations in the PIK3CA gene (36). PTEN hamartoma tumor syndrome (PHTS) caused by PTEN- or WWP1-deregulated functions $(37,38)$ could also benefit from the combinatorial treatment, which is expected to reduce the on-target toxicities of PI3K inhibition, although systemic toxicities should be assessed even more strictly in indications for these syndromes, as long-term treatment will likely be required. Thus, combinatorial PI3K and WWP1 inhibition paves the way for effective therapeutic modalities for both cancer prevention and therapy.

\section{Methods}

Cell lines and PDXs. SUM159PT, SUM149PT, and MDA-MB-231 isogenic parental and resistant cells were gifts from Alex Toker (Beth Israel Deaconess Medical Center, Harvard Medical School, Boston, Massachusetts, USA) (39). The other human and mouse cell lines used in this study were purchased from the American Type Culture Collection (ATCC; http://www.atcc.org). TNBC subtype classification was done according to Lehmann et al. (40). For PDXs, a xenograft derived from TNBC tissue (PDX TNBC) was purchased from The Jackson Laboratory (TM00089), and a xenograft derived from ER-positive breast cancer tissue (PDX ER) was a gift from Alana Welm (Huntsman Cancer Institute, University of Utah, Salt Lake City, Utah, USA).

Murine models. Wwp1 $1^{-/-}$mice and their paired $W w p 1^{+/+}$mice (on a C57BL/6J background) were obtained from Lydia Matesic (University of South Carolina, Columbia, South Carolina, USA) (41). C57BL/6J, $J: N U$ nude mice, and SCID-BEIGE mice were purchased from The Jackson Laboratory. Mouse breast cancer lysates were extracted from the primary tumor of the MMTV-PyVT mouse model.

Plasmids, reagents, and antibodies. pLenti-HA-WWP1WT-Puro, MYC-WWP1-WT, MYC-WWP1-CA, Flag-PTEN, His-ubiquitin, HA-AMPKa1, and HA-AMPKa2 were gifts from Wenyi Wei (Beth Israel 
Deaconess Medical Center, Harvard Medical School, Boston, Massachusetts, USA). Lentivirus-based constructs that expressed shRNAs targeting human WWP1 (TRCN0000003395, TRCN0000003396) and mouse WWP1 (TRCN0000028255, TRCNO000028281) were purchased from MilliporeSigma. siGENOME siRNAs for mouse Pten (M-003023-02-0005), Prkaa2 (D-040809-02-0002), and the nontargeting control (D-001210-01-05) were purchased from Horizon Discovery. pcdh-Flag-PTEN-Neo was constructed from Flag-PTEN with the In-Fusion HD Cloning Kit (Takara) according to the manufacturer's protocol. pLenti-Myc-GLUT4-mCherry (no. 64049) was purchased from Addgene. Lipofectamine 2000, RPMI-1640, DMEM, Ham's F-12, Opti-MEM reduced serum media, FBS, horse serum, and growth factor-reduced Matrigel were purchased from Thermo Fisher Scientific. Polybrene was purchased from Santa Cruz Biotechnology. Insulin, puromycin, hydrocortisone, metformin, potassium cyanide (KCN), and indole-3-carbinol (I3C) were purchased from MilliporeSigma. BKM12O and dorsomorphin (compound C) were purchased from Selleck Chemicals. BAY80-6946, BYL719, GDC-0032, and TGX-221 were purchased from MedChem Express. For in vivo treatments, BKM120 was purchased from MedKoo Biosciences. For WB, anti-PTEN (9559), anti-EGFR (4267), anti-LRP6 (2560), anti-c-Myc (D84C12, 13987), anti-Myc-tag (9B11, 2276), anti-ubiquitin (3936), anti-cleaved caspase 3 (9661), anti-p-AKT (pSer473, 9271; pThr308, 9275), anti-AKT (pan AKT, 4685), anti-pS6 (2211), anti-S6 (2217), anti-p-AMPKa (pThr172, 2535), and anti-AMPK $\alpha$ (5831) antibodies were purchased from Cell Signaling Technology. Anti-WWP1 (human) (H00011059-M01) was purchased from Novus Biologicals, anti-WWP1 (mouse; ab43791) was purchased from Abcam, and anti-HSP9O (BDB610419) was purchased from Thermo Fisher Scientific. Anti-HA-tag probe (2-2.2.14, 26183) was purchased from Thermo Fisher Scientific. Anti-6xHis (631212) was purchased from Clontech. For immunoprecipitation, anti-PTEN (A2B1, sc-7974 AC) and anti-normal mouse IgG (sc-2025) antibodies were purchased from Santa Cruz Biotechnology. PI $(3,4,5)$ P3 PIP Beads (P-B345A-2) and PI(4,5)P2 PIP Beads (P-B045A-2) were purchased from Echelon Biosciences. For ChIP-qPCR assays, anti-cMyc (D84C12, 13987) was purchased from Cell Signaling Technology. For immunohistochemical analysis, anti-Ki-67 (MA5-14520) was purchased from Thermo Fisher Scientific. For immunofluorescence staining, anti-Myc Tag DyLight 488 (MA1-21316-D488) was purchased from Thermo Fisher Scientific, and anti-PTEN (6H2.1, ABM-2052) was purchased from Cascade Bioscience.

Cell culturing. 293T, MDA-MB-231, MDA-MB-468, MCF7, CAMA1 , and undifferentiated $\mathrm{C} 2 \mathrm{C} 12$ cells were cultured in DMEM media supplemented with $10 \% \mathrm{FBS}$ and $100 \mathrm{U} / \mathrm{mL}$ penicillin and streptomycin (Invitrogen, Thermo Fisher Scientific). HCC1937 and BT549 cells were cultured in RPMI-1640 media supplemented with 10\% FBS and $100 \mathrm{U} / \mathrm{mL}$ penicillin and streptomycin. SUM159PT and SUM149PT cells were cultured in Ham's F12 media supplemented with 5\% FBS, $1 \mu \mathrm{g} / \mathrm{mL}$ hydrocortisone, $5 \mu \mathrm{g} / \mathrm{mL}$ insulin, and $100 \mathrm{U} / \mathrm{mL}$ penicillin and streptomycin. For culturing of differentiated mouse C2C12 cells, DMEM supplemented with $2 \%$ horse serum and $1 \mu \mathrm{M}$ insulin was used. All cells were incubated at $37^{\circ} \mathrm{C}, 20 \% \mathrm{O}_{2}$, and $5 \% \mathrm{CO}_{2}$.

Lentivirus production and infection. To generate recombinant lentivirus, 293T cells were cotransfected with the packaging plasmids VSVG, PMDL, and REV (purchased from Addgene, \#12259, \#12251, and \#12253) and the indicated lentivirus-based constructs. The cultured media containing virus were collected and filtered with a 0.22 $\mu \mathrm{m}$ cell strainer. For infection, the viral stock was supplemented with $10 \mu \mathrm{g} / \mathrm{mL}$ polybrene, and the infected cells were selected by $1-2 \mu \mathrm{g} /$ $\mathrm{mL}$ puromycin for at least 2 days.

Western blotting and immunoprecipitation. For Western blotting (WB), cells were lysed in RIPA buffer (Boston BioProducts) supplemented with protease (cOmplete EDTA-Free, Roche) and phosphatase (PhosStop, Roche) inhibitors. Proteins were separated on NuPAGE 4\%-12\% Bis-Tris Gradient Gels (Invitrogen, Thermo Fisher Scientific) and transferred onto polyvinylidene difluoride membranes (Immobilon-P, MilliporeSigma), and the blots were probed with the indicated antibodies. For immunoprecipitation, MDA-MB-231 cells with stable expression of the indicated vectors were treated with DMSO or BKM120 for the indicated durations and subsequently lysed in RIPA buffer with protease and phosphatase inhibitors. Total lysates $(24 \mu \mathrm{g})$ were precleared for 30 minutes at $4^{\circ} \mathrm{C}$ and then immunoprecipitated with anti-PTEN (1:100) antibody or anti-mouse IgG antibody (1:100) overnight at $4^{\circ} \mathrm{C}$. The protein $\mathrm{G}$ sepharose beads (GE Healthcare) were then added and incubated for another 2 hours. The immunoprecipitates were washed with RIPA buffer 3 times and denatured with Reducing Laemmli Buffer (Boston BioProducts). Samples were separated on NuPAGE $4 \%-12 \%$ Bis-Tris Gradient Gels (Invitrogen, Thermo Fisher Scientific). Quantitation of the intensity of the blot was performed using Fiji software (ImageJ, NIH).

Drug sensitivity assay. To determine the $\mathrm{IC}_{50}$ value, $6.0 \times 10^{4}$ of cells with stably expressing shWWP1 were treated with stepwise concentrations of BKM120 or I3C for 5 days with media refreshed every 2 days. Cells were fixed with $10 \%$ formalin followed by staining with $0.1 \%$ crystal violet. It was then solubilized with $10 \%$ acetic acid, and the absorbance was measured with a SpectraMax iD3 (Molecular Devices). The normalized measurements were used to obtain dose response curves and $\mathrm{IC}_{50}$ values. Drug synergism studies were carried out using Combenefit Software (Cancer Research UK Cambridge Institute) (42).

Subcellular fractionation. Membrane and cytosolic fractionation of MDA-MB-231 cells transduced with the indicated constructs was performed using the Subcellular Protein Fraction Kit for Cultured Cells (Thermo Fisher Scientific) according to the manufacture's procedures. Briefly, $2.0 \times 10^{5}$ MDA-MB-231 or MCF7 cells were seeded in a $6 \mathrm{~cm}$ plate and treated with $1.0 \mu \mathrm{M}$ BKM120 for the indicated durations. Cells were immediately harvested and processed to extract each subcellular fraction.

Immunofluorescence cytochemistry. MDA-MB-231 cells were seeded onto 4-chamber slide glass (Iwaki Cell Biology) and treated with BKM120 for 12 hours. After a 3-hour serum starvation followed by 10 minutes of insulin stimulation $(0.5 \mu \mathrm{g} / \mathrm{mL})$, cells were fixed with $4 \%$ paraformaldehyde for 10 minutes at room temperature followed by permeabilization with $0.5 \%$ Triton X. Subsequently, cells were incubated with anti-PTEN antibody diluted in Can Get Signal Immunostain Solution A (1:100, Toyobo) for 1 hour at room temperature followed by incubation with goat anti-rabbit IgG H\&L (Alexa Fluor 488, Thermo Fisher Scientific). Slides were sealed by coverglasses with VECTASHIELD Mounting Medium with DAPI (Vector Laboratories) (10, 43).

Generation of isogenic cells resistant to BKM120. Isogenic resistant MDA-MB-231 and MCF7 cells were generated by repeated exposure to stepwise increasing concentrations of BKM120 over a period of several months. The dose of BKM120 was increased stepwise once cells were recovered to achieve a sufficient growth rate compared with that of parental cells. The resistant cells generated were maintained with the 
media containing the following doses of BKM120: 1.0 $\mu \mathrm{M}$ for resistant MB-231 LD cells; $1.5 \mu \mathrm{M}$ for resistant MB-231 HD cells; $0.5 \mu \mathrm{M}$ for resistant MCF7 low-dose (LD) cells; $0.75 \mu \mathrm{M}$ for resistant MCF7 middle-dose (MD) cells; and 1.0 $\mu \mathrm{M}$ for resistant MCF7 HD cells, respectively.

RNA isolation and quantitative PCR. Total RNA was purified from cell lines using the PureLink RNA Mini Kit (Invitrogen, Thermo Fisher Scientific). For quantitative PCR (qPCR) analysis, $1.0 \mu$ g total RNA was reverse transcribed into cDNA using the SuperScript IV First Strand Synthesis System (Thermo Fisher Scientific). SYBR-Green qPCR analysis was performed with PowerUp SYBR Green Master Mix (Thermo Fisher Scientific) by means of StepOnePlus (Applied Biosystems), in accordance with the manufacturer's protocol. The following primers were used: human WWP1, forward, 5'-TGCTTCACCAAGGTCTGATACT-3', reverse, 5'-GCTGTTCCGAACCAGTTCTTTT3'; ACTB, forward, 5'-CTGTGCTACGTCGCCCTGG-3', reverse, 5'-GGCTGTATTCCCCTCCATCG-3'; human AMPK $\alpha 1$, forward, 5'-AGGAAGAATCCTGTGACAAGCAC-3', reverse, 5'-CCGATCTCTGTGGAGTAGCAGT-3'; AMPK $\alpha 2$, forward, 5' -CCAGAGAATGTCCTGTTGGATGC-3', reverse, 5'-CTGAGATGACTTCAGGTGCTGC-3'; mouse AMPK $\alpha 1$, forward, 5'-CTCAGTTCCTGGAGAAAGATGG-3', reverse, 5'-CTGCCGGTTGAGTATCTTCAC-3'; AMPK $\alpha 2$, forward, 5'-CATGGCTGAGAAGCAGAAGCAC-3', reverse, 5'-CTTAACTGCCACTTTATGGCCTG-3'; mouse ACTB, forward, 5'-GGCTGTATTCCCCTCCATCG-3', reverse, 5' -CCAGTTGGTAACAATGCCATGT-3'.

ChIP-qPCR assay. ChIP assays were performed with MDAMB-231 parental or resistant cells using the SimpleChIP Enzymatic Chromatin IP Kit (Cell Signaling Technology) according to the manufacturer's protocol and a previous report (10). Briefly, $6.0 \times 10^{6}$ cells were crosslinked with formaldehyde, and nuclear extraction was treated with micrococcal nuclease for 20 minutes at $37^{\circ} \mathrm{C}$. Chromatin fractions were incubated with $10 \mathrm{mg}$ anti-H3 (provided in the kit), antiMyc antibody, or normal IgG at $4^{\circ} \mathrm{C}$ overnight with Magnetic Protein $\mathrm{G}$ Beads. After extensive washing, samples were reverse-crosslinked, and immunoprecipitated DNAs were purified with DNA purification collection columns. Samples were subjected to qPCR with Tunderbird SYBR qPCR Mix (Toyobo). Myc-binding sites in gene promoter regions were determined by the ENCODE database of ChIP-Seq analyses (ENCSRO0ODMQ; https://www.encodeproject.org/chipseq/transcription factor/). The following primers were used: WWP1, forward, 5'-GGGAGGGTCGGGTGTC -3', reverse, 5'-CTTCCGCGCCCAAGAT-3'; JunB, forward, 5'-AAGCCCACAGAGAGAGGTGGAAG-3', reverse, 5'-CCAGAAGGTGGTGCCTTTTTATTG-3'.

Glucose and insulin measurement. For $W w p 1^{-/-}$mice and the paired $W_{w p 1^{+/+}}$mice, 6- to 8-week-old female mice were treated with $0.5 \%$ carboxymethyl cellulose (CMC) (MilliporeSigma) as a vehicle control or $50 \mathrm{mg} / \mathrm{kg}$ BKM120, and peripheral blood was collected from the tail of mice every 30 minutes and subjected to glucose measurement with Germaine Laboratories AimStrip Plus Blood Glucose Testing System (Thermo Fisher Scientific). Blood insulin was measured 3 hours after BKM120 administration with the Ultra Sensitive Mouse Insulin ELISA Kit (Crystal Chem). For combination therapy experiments with C58BL6J mice, 6- to 8-week-old male mice were pretreated intraperitoneally with $\mathrm{I} 3 \mathrm{C}(50 \mathrm{mg} / \mathrm{kg})$ or metformin $(200 \mathrm{mg} /$ $\mathrm{kg}$ ) dissolved in 5\% DMSO, every 2 days for 1 week. The mice were then administrated BKM120 (50 mg/ kg) by oral gavage, followed by measurement over time of blood glucose and insulin levels. For the glucose assay with the AMPK inhibitor, $20 \mathrm{mg} / \mathrm{kg}$ CC or PBS was administrated intraperitoneally to $W w p 1^{-/-}$and $W w p 1^{+/+}$mice 30 minutes before BKM120 treatment $(50 \mathrm{mg} / \mathrm{kg}$, oral), followed by sequential measurement of blood glucose over a 3-hour period.

Mouse tissue extraction. For extraction of mouse muscle tissues, 6- to 8-week-old female $\mathrm{Wwp1}^{-/-}$and $\mathrm{Wwp1}^{+/+}$mice were treated with $0.5 \%$ CMC or BKM120 $(50 \mathrm{mg} / \mathrm{kg})$ following a 4-hour starvation. Mice were euthanized 2 hours after treatment, and the quadriceps muscle was then extracted and lysed in RIPA buffer for WB.

In vivo ubiquitination assay. To analyze in vivo ubiquitination of AMPK $\alpha$, cells were transfected with His-ubiquitin, Myc-WWP1, and HA-AMPK $\alpha$. Cells were lysed by buffer A ( $6 \mathrm{M}$ guanidine- $\mathrm{HCl}, 50 \mathrm{mM}$ $\mathrm{Na}_{2} \mathrm{HPO}_{4} / \mathrm{NaH}_{2} \mathrm{PO}_{4}[\mathrm{pH} 8.0]$, and $10 \mathrm{mM}$ imidazole), and lysates were incubated with Ni-NTA agarose for 1.5 hours at $4^{\circ} \mathrm{C}$. The beads were washed once with buffer A, twice with buffer A/TI ( 1 vol buffer A/3 vol buffer TI [ $25 \mathrm{mM}$ Tris- $\mathrm{HCl}, \mathrm{pH} 6.8$, and $20 \mathrm{mM}$ imidazole]), and 3 times with buffer TI, and then analyzed by WB. In all experiments, an equal amount of His-ubiquitin expression was verified by WB.

Quantitation of surface GLUT4. C2C12 cells with stable expression of Myc-GLUT4-mCherry were generated and sorted by mCherry via flow cytometry (C2C12-Myc-GLUT4-mCherry). The cells were transduced with shWWP1 lentiviral particles and treated with 2.0 $\mu \mathrm{M}$ BKM120 for 16 hours with serum starvation. After the stimulation with $100 \mathrm{nM}$ insulin for 15 minutes, cells were treated with 2.0 $\mathrm{mM} \mathrm{KCN}$ at $4^{\circ} \mathrm{C}$ for 5 minutes to avoid subsequent internalization of GLUT4 and then harvested. The cells were incubated with anti-Myctagged Dylight 488 antibody at $4^{\circ} \mathrm{C}$ for 30 minutes and subjected to flow cytometry. The MFI of Myc-tag and mCherry was analyzed using FlowJo software (BD). The surface GLUT4 ratio was determined by dividing the MFI of Myc-tag by that of mCherry $(12,44,45)$.

Glucose uptake assay. Glucose uptake was measured with a Glucose Uptake-Glo Assay Kit (Promega) according to the manufacturer's protocol. Briefly, differentiated $\mathrm{C} 2 \mathrm{C} 12$ cells with stable expression of shWWP1 $\left(7.5 \times 10^{4}\right.$ cells) were seeded in 24-well plates, and $25 \mathrm{nM}$ siAMPK, siPTEN, or a nontargeting control (siControl) was transfected into the cells with DharmaFECT 1 Transfection Reagent (Horizon Discovery). The cells were treated with $2.0 \mu \mathrm{M}$ BKM120 for 2 hours after overnight serum starvation. After the stimulation with $100 \mathrm{nM}$ insulin for 15 minutes, cells were treated with $1.0 \mathrm{mM}$ 2-deoxy-D-glucose (DG) for 10 minutes and subsequently reacted with 2-deoxyglucose-6-phosphate (2DG6P) detection reagent. Luciferase activity was measured with a GloMax 96 Microplate Luminometer (Promega).

Measurement of ADP/ATP ratio. In a 96-well plate, $5.0 \times 10^{3}$ C2C12 cells stably expressing a control shRNA or $W w p 1$ were seeded and treated with BKM120 for 2 hours. The ADP/ATP ratio was measured using the ADP/ATP ratio assay kit (MilliporeSigma) according to the manufacturer's protocol.

Soft agar colony formation assay. In 6-well plates, $2 \mathrm{~mL}$ bottom layer medium (DMEM and 10\% FBS with $0.6 \%$ agarose) was added to each well and cooled to $4^{\circ} \mathrm{C}$ for 10 minutes. Then, $2 \mathrm{~mL}$ top layer medium (DMEM and 10\% FBS with $0.3 \%$ agarose) mixed with $1.0 \times$ $10^{4}$ of MDA-MB-231 cells was added to each well and cooled to room temperature for 20 minutes. Cells were treated with BKM120 (1.0 $\mu \mathrm{M})$; BAY80-6946 (0.5 $\mu \mathrm{M})$; BYL719 $(3.0 \mu \mathrm{M})$; GDC-0032 $(1.0 \mu \mathrm{M})$; TGX-221 $(3.0 \mu \mathrm{M})$; or I3C $(100 \mu \mathrm{M})$ and cultured at $37^{\circ} \mathrm{C}$ for 28 days. To visualize colonies, $1 \mathrm{~mL} 0.005 \%$ crystal violet was added to each well for 1 hour at room temperature. Colonies were counted with Fiji software. Each experiment was performed in triplicate. 
Xenotransplantation of cell lines and PDX models. To assay tumor growth in the xenograft model of cell lines, 6-week-old female J:NU nude mice housed in specific pathogen-free environments were subcutaneously inoculated in the flank with $0.5 \times 10^{6}$ MDA-MB-231 parental or resistant cells mixed with an equal volume of DMEM medium and Matrigel. Mice were administrated BKM120 (30 mg/kg) or I3C (100 $\mathrm{mg} / \mathrm{kg}$ ), which was resuspended in $0.5 \% \mathrm{CMC}$ via oral gavage in $150 \mu \mathrm{L}$ every day. The mice were euthanized when the largest tumor reached $2000 \mathrm{~cm}^{2}$ in size. For MCF7 xenografts, $2.0 \times 10^{6}$ of cells were transplanted subcutaneously into the mice. Mice were orally treated with BYL719 $(20 \mathrm{mg} / \mathrm{kg})$ or I3C $(100 \mathrm{mg} / \mathrm{kg}) 5$ time a week for 7 weeks and subsequently euthanized. For PDX models, the surgically excised tumor from the parental mouse was cut into small $(2-4 \mathrm{~mm})$ pieces and inoculated subcutaneously into the dorsal region of the trunk of 6-week-old female SCID-BEIGE mice. For mice with ER-positive PDX ER tumors, a $17 \beta$-estradiol pellet $(0.72 \mathrm{mg}$, Innovative Research of America) was subcutaneously implanted into the lateral side of the neck. Five times a week, mice were administrated BYL719 $(20 \mathrm{mg} / \mathrm{kg})$ or I3C $(200 \mathrm{mg} /$ $\mathrm{kg}$ ), which was resuspended in $0.5 \% \mathrm{CMC}$ via oral gavage in $150 \mu \mathrm{L}$.

Immunohistochemical analysis for mouse tissues. Individual xenograft tumors derived from nude mice were dissected and fixed in $4 \%$ paraformaldehyde and subsequently embedded in paraffin according to standard procedures. Slides were processed for staining with antiKi67 antibody (1:200). The stained slides were visualized by a brightfield microscope (Nikon Eclipse 50i).

Statistics. Statistical analysis was performed using 1-way ANOVA or repeated-measures, 2-way ANOVA followed by Tukey-Kramer multiple-comparison test for multigroup data sets, or by unpaired 2-tailed Student's $t$ tests for comparison of 2 groups. Tumor size was analyzed using linear mixed-effects model regression to analyze the repeated measurements on each tumor. $P$ values of less than 0.05 were considered statistically significant. All statistical tests were executed using the R statistical software program, version 3.6.3 (https://www.r-project.org/) or Excel (Microsoft).
Study approval. The care and treatment of animals were approved by the IACUC of Beth Israel Deaconess Medical Center (protocols 076-2017 and 054-2020).

\section{Author contributions}

TK, JGC, YRL, and PPP conceived and designed the study and experiments. TK, YRL, HH, GN, and LW performed the experiments and data analyses. MO, KK, AT, WW, YRL, and PPP supervised the study. TK, HH, LW, NP, VM, SB, and SL performed mouse experiments. LEM provided the $W w p 1^{1^{-/}}$mice and the paired $W w p 1^{1^{+/}}$mice. GMW provided the PDX tumor model. TK, JGC, YRL, and PPP interpreted the data and wrote the manuscript. All authors revised, critically discussed, and approved the final version of the manuscript.

\section{Acknowledgments}

We thank Jean Zhao for sharing cell lines with our team. We are also grateful to all the members of the Pandolfi laboratory for their constructive criticism, as well as to T. Garvey for editing the manuscript. This work was supported by NIH grants R01 CA82328 and R35 CA197529 (to PPP). TK was supported by a Japan Society for the Promotion of Science (JSPS) Overseas Research Fellowship, a Research Fellowship of the Uehara Memorial Foundation, and Grants-in-Aid from the Ministry of Education, Culture, Sports, Science, and Technology of Japan (21H02893). YRL was supported in part by a Postdoctoral Research Abroad Program Fellowship, the Taiwan National Science Council (NSC), and a DOD Prostate Cancer Research Program (PCRP) Postdoctoral Training Award (W81XWH-16-1-0249). HH was supported by a JSPS Overseas Research Fellowship.

Address correspondence to: Pier Paolo Pandolfi, Renown Institute for Cancer, Nevada System of Higher Education, 1155 Mill Street, Reno, Nevada 89502, USA.Email: PierPaolo.PandolfiDeRinaldis@ renown.org.
1. Fruman DA, et al. The PI3K pathway in human disease. Cell. 2017;170(4):605-635.

2. Engelman JA, et al. The evolution of phosphatidylinositol 3-kinases as regulators of growth and metabolism. Nat Rev Genet. 2006;7(8):606-619.

3. Hennessy BT, et al. Exploiting the PI3K/AKT pathway for cancer drug discovery. Nat Rev Drug Discov. 2005;4(12):988-1004.

4. Mayer IA, Arteaga CL. The PI3K/AKT pathway as a target for cancer treatment. Annu Rev Med. 2016;67:11-28.

5. Lee YR, et al. The functions and regulation of the PTEN tumour suppressor: new modes and prospects. Nat Rev Mol Cell Biol. 2018;19(9):547-562.

6. Song MS, et al. The functions and regulation of the PTEN tumour suppressor. Nat Rev Mol Cell Biol. 2012;13(5):283-296.

7. Papa A, Pandolfi PP. The PTEN-PI3K axis in cancer. Biomolecules. 2019;9(4):153.

8. Dillon LM, Miller TW. Therapeutic targeting of cancers with loss of PTEN function. Curr Drug Targets. 2014;15(1):65-79.

9. Juric D, et al. Convergent loss of PTEN leads to clinical resistance to a PI(3)K $\alpha$ inhibitor. Nature. 2015;518(7538):240-244.
10. Lee YR, et al. Reactivation of PTEN tumor suppressor for cancer treatment through inhibition of a MYC-WWP1 inhibitory pathway. Science. 2019;364(6441):eaau0159.

11. Trotman LC, et al. Ubiquitination regulates PTEN nuclear import and tumor suppression. Cell. 2007;128(1):141-156.

12. Costa C, et al. Measurement of PIP3 levels reveals an unexpected role for $\mathrm{p} 110 \beta$ in early adaptive responses to $\mathrm{p} 110 \alpha$-specific inhibitors in luminal breast cancer. Cancer Cell. 2015;27(1):97-108.

13. Yang J, et al. Targeting PI3K in cancer: mechanisms and advances in clinical trials. Mol Cancer. 2019;18(1):26.

14. Dey N, et al. MYC-xing it up with PIK3CA mutation and resistance to PI3K inhibitors: summit of two giants in breast cancers. Am JCancer Res. 2015;5(1):1-19.

15. Liu P, et al. Oncogenic PIK3CA-driven mammary tumors frequently recur via PI3K pathwaydependent and PI3K pathway-independent mechanisms. Nat Med. 2011;17(9):1116-1120.

16. Dienstmann R, et al. Picking the point of inhibition: a comparative review of PI3K/AKT/ mTOR pathway inhibitors. Mol Cancer Ther.
2014;13(5):1021-1031.

17. Andre F, et al. Alpelisib for PIK3CA-mutated, hormone Receptor-positive advanced breast cancer. N Engl J Med. 2019;380(20):1929-1940.

18. Di Leo A, et al. Buparlisib plus fulvestrant in postmenopausal women with hormone-receptorpositive, HER2-negative, advanced breast cancer progressing on or after mTOR inhibition (BELLE-3): a randomised, double-blind, placebocontrolled, phase 3 trial. Lancet Oncol. 2018;19(1):87-100.

19. Baselga J, et al. Buparlisib plus fulvestrant versus placebo plus fulvestrant in postmenopausal, hormone receptor-positive, HER2-negative, advanced breast cancer (BELLE-2): a randomised, double-blind, placebo-controlled, phase 3 trial. Lancet Oncol. 2017;18(7):904-916.

20. Martin M, et al. A randomized adaptive phase II/ III study of buparlisib, a pan-class I PI3K inhibitor, combined with paclitaxel for the treatment of HER2- advanced breast cancer (BELLE-4). Ann Oncol. 2017;28(2):313-320.

21. Knowler WC, et al. Reduction in the incidence of type 2 diabetes with lifestyle intervention or metformin. NEngl JMed. 2002;346(6):393-403. 
22. Foretz M, et al. Metformin inhibits hepatic gluconeogenesis in mice independently of the LKB1/ AMPK pathway via a decrease in hepatic energy state. JClin Invest. 2010;120(7):2355-2369.

23. Lee JO, et al. E3 ubiquitin ligase, WWP1, interacts with AMPK $\alpha 2$ and down-regulates its expression in skeletal muscle C2C12 cells. J Biol Chem. 2013;288(7):4673-4680.

24. Deng $M$, et al. Deubiquitination and activation of AMPK by USP10. Mol Cell. 2016;61(4):614-624.

25. Al-Maghrabi J, et al. AMPK expression patterns are significantly associated with poor prognosis in breast cancer patients. Ann Diagn Pathol. 2017;29:62-67.

26. Fox MM, et al. AMP-Activated protein kinase $\alpha$ 2 isoform suppression in primary breast cancer alters AMPK growth control and apoptotic signaling. Genes Cancer. 2013;4(1-2):3-14.

27. Cheng J, et al. AMP-activated protein kinase suppresses the in vitro and in vivo proliferation of hepatocellular carcinoma. PLoS One. 2014;9(4):e93256.

28. Russell RR, et al. Translocation of myocardial GLUT-4 and increased glucose uptake through activation of AMPK by AICAR. Am J Physiol. 1999;277(2):H643-H649.

29. Manning BD, Toker A. AKT/PKB signaling: navi- gating the network. Cell. 2017;169(3):381-405.

30. Lim CY, et al. Tropomodulin 3 is a novel Akt2 effector regulating insulin-stimulated GLUT4 exocytosis through cortical actin remodeling. Nat Commun. 2015;6:5951.

31. Garrido-Castro AC, et al. Phase 2 study of buparlisib (BKM120), a pan-class I PI3K inhibitor, in patients with metastatic triple-negative breast cancer. Breast Cancer Res. 2020;22(1):120.

32. Cancer Genome Atlas Network. Comprehensive molecular portraits of human breast tumours. Nature. 2012;490(7418):61-70.

33. Hopkins BD, et al. Suppression of insulin feedback enhances the efficacy of PI3K inhibitors. Nature. 2018;560(7719):499-503.

34. Aggarwal BB, Ichikawa H. Molecular targets and anticancer potential of indole-3-carbinol and its derivatives. Cell Cycle. 2005;4(9):1201-1215.

35. Bradlow HL, et al. Long-term responses of women to indole-3-carbinol or a high fiber diet. Cancer Epidemiol Biomarkers Prev. 1994;3(7):591-595.

36. Venot $Q$, et al. Targeted therapy in patients with PIK3CA-related overgrowth syndrome. Nature. 2018;558(7711):540-546.

37. Lee YR YL, et al. WWP1 gain-of-function triggers PTEN inactivation to drive cancer predisposition. N Engl J Med. 2020;382(22):2103-2116.
38. Yehia L, et al. PTEN-opathies: from biological insights to evidence-based precision medicine. JClin Invest. 2019;129(2):452-464.

39. Clement E, et al. Skp2-dependent reactivation of AKT drives resistance to PI3K inhibitors. Sci Signal. 2018;11(521):eaao3810.

40. Lehmann BD, et al. Identification of human triple-negative breast cancer subtypes and preclinical models for selection of targeted therapies. JClin Invest. 2011;121(7):2750-2767.

41. Zhao L, et al. Tumor necrosis factor inhibits mesenchymal stem cell differentiation into osteoblasts via the ubiquitin E3 ligase Wwp1. Stem Cells. 2011;29(10):1601-1610.

42. Di Veroli GY, et al. Combenefit: an interactive platform for the analysis and visualization of drug combinations. Bioinformatics. 2016;32(18):2866-2868.

43. Papa A, et al. Cancer-associated PTEN mutants act in a dominant-negative manner to suppress PTEN protein function. Cell. 2014;157(3):595-610.

44. Lampson MA, et al. Demonstration of insulinresponsive trafficking of GLUT4 and vpTR in fibroblasts. JCell Sci. 2000;113(pt 22):4065-4076.

45. Camus SM, et al. CHC22 clathrin mediates traffic from early secretory compartments for human GLUT4 pathway biogenesis. J Cell Biol. 2020;219(1):e201812135. 